SFB

On confidence bands for

823

multivariate nonparametric regression

Katharina Proksch

Nr. 1/2014

S

$\mathcal{O}$

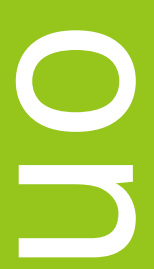

0

(1)

(D)

SFB
823
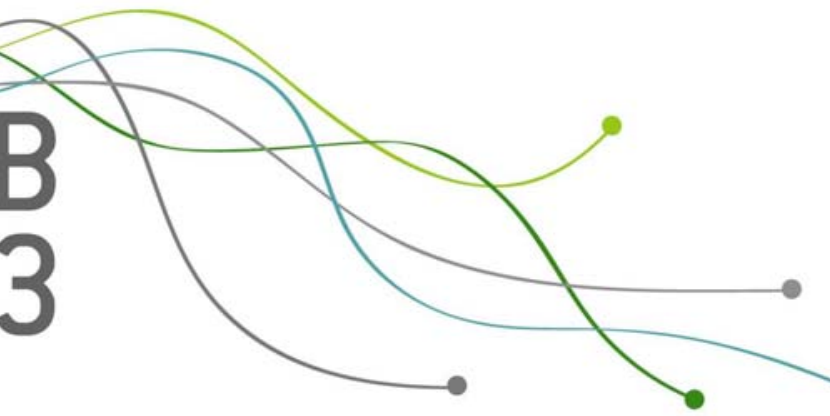



\title{
On Confidence bands for multivariate nonparametric regression
}

\author{
Katharina Proksch \\ Ruhr-Universität Bochum \\ Fakultät für Mathematik \\ 44780 Bochum, Germany \\ e-mail: katharina.proksch@rub.de
}

December 6, 2013

\begin{abstract}
In a multivariate nonparametric regression problem with fixed, deterministic design asymptotic, uniform confidence bands for the regression function are constructed. The construction of the bands is based on the asymptotic distribution of the maximal deviation between a suitable nonparametric estimator and the true regression function which is derived by multivariate strong approximation methods and a limit theorem for the supremum of a stationary Gaussian field over an increasing system of sets. The results are derived for a general class of estimators which includes local polynomial estimators as a special case. The finite sample properties of the proposed asymptotic bands are investigated by means of a small simulation study.
\end{abstract}

Keywords and Phrases: Confidence bands, Rates of convergence, Multivariate regression, Nonparametric Regression, Uniform convergence.

AMS Subject Classification: 62E20, 62G08

\section{Introduction}

Within the last decades nonparametric regression has received a great deal of attention as a powerful tool for data analysis. Various different models and methods have been discussed and thoroughly investigated. Nonparametric curve estimation provides many useful applications, especially for graphical visualization but it can also serve as basis for the development of means of statistical inference such as goodness of fit 
tests or the construction of confidence sets for the unknown regression function. While interval estimates can be used for its point-wise analysis, simultaneous confidence bands have to be employed in order to draw conclusions regarding global features of the curve under consideration and thus shed more light into the connection between dependent and independent variables.

In this paper we develop new asymptotic uniform confidence sets in a nonparametric regression setting with a deterministic and multivariate predictor. To be precise we consider the multivariate regression model

$$
Y_{\mathbf{i}}=f\left(\mathbf{t}_{\mathbf{i}}\right)+\varepsilon_{\mathbf{i}}, \mathbf{i}:=\left(i_{1}, \ldots, i_{d}\right) \in\{1, \ldots, n\}^{d},
$$

where $\mathbf{i}=\left(i_{1}, \ldots, i_{d}\right)$ is a multi-index, the $\left\{\mathbf{t}_{\mathbf{i}}:=\left(t_{i_{1}}, \ldots, t_{i_{d}}\right) \in \mathbb{R}^{d} \mid 1 \leq i_{1}, \ldots, i_{d} \leq n\right\}$ are deterministic design points in $\mathbb{R}^{d},\left\{\varepsilon_{\left(i_{1}, \ldots, i_{d}\right)} \mid 1 \leq i_{1}, \ldots, i_{d} \leq n\right\}$ is a field of centered, independent identically distributed random variables with common variance $\sigma^{2}$ and $f$ is an unknown, smooth regression function. The construction of confidence sets requires a reliable estimate of the unknown object. Often kernel smoothing techniques are applied in this context (cf., e.g., Wand and Jones (1995)). Alternative approaches, such as spline smoothing for instance, often show similar asymptotic behaviour in the sense that corresponding estimators have approximately the same form, that is, linear in the observations and with a kernel that is of convolution form and possibly variable with respect to the sample size $n$.

Given a suitable estimate, a well-established method to construct asymptotic uniform confidence bands is based on the original work of Bickel and Rosenblatt (1973b) who extended results of Smirnov (1950) for a histogram estimate and constructed confidence bands for a univariate density function of independent identically distributed observations. Their method is based on the asymptotic distribution of the supremum of a centered kernel density estimator and closely related to extreme value theory. Since this seminal paper the idea has been elaborated, advanced and adopted to various situations. For example Johnston (1982) constructed confidence bands based on the Nadaraya-Watson and Yang estimator, Härdle (1989) derived asymptotic uniform confidence bands for $M$-smoothers. Eubank and Speckman (1993), who considered deterministic, uniform design and local constant estimation, and Xia (1998), who considered random design points under dependence and local linear estimation, employed an explicit bias-correction. Bootstrap confidence bands for nonparametric regression were proposed by Neumann and Polzehl (1998) and Claeskens and van Keilegom (2003). Härdle and Song (2010) investigated asymptotic 
uniform confidence bands for a quantile regression curve with a one-dimensional predictor. In the context of density estimation Giné et al. (2004) derived asymptotic distributions of weighted suprema. Further, confidence bands were proposed in adaptive density estimation based on linear wavelet and kernel density estimators (Giné and Nickl (2010)), density deconvolution (Bissantz et al. (2007)) or adaptive density deconvolution (Lounici and Nickl (2011)). All these authors, if not otherwise indicated, employed undersmoothing in order to cope with the bias. Also, in all the above listed references one-dimensional models are considered and the results are not applicable in cases where the quantity of interest depends on a multivariate predictor. On the other hand only a few results can be found in a multivariate setting which attracted comparatively little attention so far. For instance, in the same year the well-known paper Bickel and Rosenblatt (1973b) was released also a multivariate extension was published (Bickel and Rosenblatt (1973a)) which received by far less attention. Rosenblatt (1976) studied maximal deviations of multivariate density estimates, Konakov and Piterbarg (1984) investigated the convergence of the distribution of the maximal deviation for the Nadaraya-Watson estimate in a multivariate, random design regression setting and Rio (1994) investigated local invariance principles in the context of density estimation. An alternative approach was recently proposed by Hall and Horowitz (2013) who addressed the bias-difficulty explicitly and constructed confidence bands based on normal approximations and a bootstrap method that is used to adjust the level $\alpha$ in the normal quantiles in such a way that a coverage of a desired value of at least $1-\alpha_{0}$ is attained at at least a predifined portion of values $x \in \mathcal{R}$, where $\mathcal{R} \subset \mathbb{R}^{d}$. They discuss both nonparametric density and regression estimation.

In this paper we construct asymptotic uniform confidence bands for a regression function in a multivariate setting for a general class of nonparametric estimators of the regression function. For the sake of a transparent notation we focus on local polynomial estimators. However our approach is generally applicable for several other estimators in use (see Theorem 3 and Remark 2 below).

Notations and definitions as well as assumptions, required for the asymptotic theory, can be found in Section 2. For a clear exposition we examine in Section 3 the two-dimensional case, briefly discuss the properties of the estimator and state the main results. The general case of a $d$-dimensional predictor is discussed in Section 4. The finite sample properties of the proposed asymptotic bands are investigated in Section 5 and detailed proofs for the two-dimensional case are given in Section 6 while the case $d>2$ is considered in Section 7. Our arguments heavily rely on results by Piterbarg (1996) who provided a limit 
theorem for the supremum

$$
\sup _{t \in T_{n}}|X(t)|
$$

of a stationary Gaussian field $\left\{X(t) \mid t \in \mathbb{R}^{d}\right\}$, where $\left\{T_{n} \subset \mathbb{R}^{d}\right\}_{n \in \mathbb{N}}$ is an increasing system of sets such that $\lambda^{d}\left(T_{n}\right) \rightarrow \infty$ as $n \rightarrow \infty$ and also on multivariate strong approximation methods provided by Rio (1993).

\section{General setup and assumptions}

Let $\Omega:=(0,1)^{d}$ and suppose that for two positive constants $k \in \mathbb{N}$ and $a \in(0,1)$ the function $f: \bar{\Omega} \rightarrow \mathbb{R}$ from model (1) belongs to the Hölder class of functions $C^{k, a}(\bar{\Omega})$, i.e., for all multi-indices $\beta=\left(\beta_{1}, \ldots, \beta_{d}\right)$ with $|\boldsymbol{\beta}|=\beta_{1}+\ldots+\beta_{d} \leq k$ the derivatives $D^{\beta} f$ exists and $\|f\|_{C^{k, a}}<\infty$. Here we use the following notation

$$
D^{\beta} f\left(x_{1}, \ldots, x_{d}\right)=\frac{\partial^{|\beta|} f}{\partial x_{1}^{\beta_{1}} \ldots \partial x_{d}^{\beta_{d}}}\left(x_{1}, \ldots, x_{d}\right)
$$

and

$$
\|f\|_{C^{k, a}}=\max _{|\boldsymbol{\beta}| \leq k} \sup _{\mathbf{x} \in \Omega}\left|D^{\boldsymbol{\beta}} f(\mathbf{x})\right|+\max _{|\boldsymbol{\beta}|=k} \sup _{\mathbf{x}, \mathbf{y} \in \Omega, \mathbf{x} \neq \mathbf{y}} \frac{|f(\mathbf{x})-f(\mathbf{y})|}{\|\mathbf{x}-\mathbf{y}\|^{a}}<\infty
$$

where $\|\cdot\|$ without a subscript denotes the Euclidean distance. Also, in what follows, more of the usual multi-index notation will be used, such as

$$
\mathbf{u}^{\alpha}:=u_{1}^{\alpha_{1}} \cdot \ldots \cdot u_{d}^{\alpha_{d}} \quad \text { and } \quad \alpha !:=\alpha_{1} ! \cdot \ldots \cdot \alpha_{d} !
$$

Further, with a slight abuse of notation, we shall denote the vector $\left(\frac{t_{1}-x_{1}}{h_{1}}, \ldots, \frac{t_{d}-x_{d}}{h_{d}}\right)^{T}$ by $\frac{\mathbf{t}_{\mathbf{i}}-\mathbf{x}}{\mathbf{h}}$ for the sake of brevity.

Assumption 1. Assume that the following three conditions hold

(i) The kernel $K$ has compact support: $\operatorname{supp}(K) \subset[-1,1]^{d}$. 
(ii) There exist constants $D, K_{1}>0$ and $K_{2}<\infty$ such that

$$
K_{1} \cdot I_{[-D, D]^{d}}(\mathbf{u}) \leq K(\mathbf{u}) \leq K_{2} \cdot I_{[-1,1]^{d}}(\mathbf{u})
$$

(iii) All derivatives of $K$ up to the order d exist and are continuous.

Assumption 2. Suppose that the design points $\left\{t_{\mathbf{i}}=\left(t_{i_{1}}, \ldots, t_{i_{d}}\right) \mid \mathbf{i}=\left(i_{1}, \ldots, i_{d}\right) \in\{1, \ldots, n\}^{d}\right\}$ satisfy

$$
\frac{i_{j}}{n+1}=\int_{0}^{t_{i_{j}}} g_{j}(z) d z=: G_{j}\left(t_{i_{j}}\right), \quad j=1, \ldots, d
$$

for positive design densities $g_{j}, j=1, \ldots, d$ on $[0,1]$ (see also Sacks and Ylvisaker (1970)) that are bounded away from zero and continuously differentiable with bounded derivatives up to order $(d-1) \vee 1$.

Remark 1. A further generalization of the design in the sense that Assumption 2 only holds approximately, such as proposed in Dette and Munk (1998), i.e.,

$$
\max _{i=2}^{n}\left|\int_{t_{i_{j-1}}}^{t_{i_{j}}} g_{j}(t) d t-\frac{1}{n}\right|=o\left(\frac{1}{n \log (n)}\right)
$$

is also possible but will not be considered here. The additional factor of $1 / \log (n)$ is needed in order to compensate the additional factor in the convergence rate of the maximal deviation of $f$ and $\hat{f}$. Under the more general assumption (5) the rate in assertion $(i)$ of Lemma 1 would change to $o(1 / \log (n))$ and for the rate in the proof of Lemma 7 , equation (13), we would obtain $o_{P}(1 / \sqrt{\log (n)})$ instead of $O_{P}\left(\left(h_{1}+h_{2}\right) \log (n)\right)$.

\section{Bivariate nonparametric regression}

\subsection{Notation and auxiliary results}

In the following we shall adapt the notation introduced in Tsybakov (2009), Chapter 1.6 to the two dimensional setting. We shall also make use of some of the results stated therein and extend the proofs, if necessary to the case where the design only meets Assumption 2, i.e., is not necessarily uniform. In order to define the estimator we need to fix some notation first. For $j=1, \ldots, k$ let $U_{j}: \mathbb{R}^{2} \rightarrow \mathbb{R}^{j+1}$ be defined 


$$
U_{j}(\mathbf{u}):=\left(\frac{\mathbf{u}^{(j, 0)}}{j !}, \frac{\mathbf{u}^{(j-1,1)}}{(j-1) ! \cdot 1 !}, \frac{\mathbf{u}^{(j-2,2)}}{(j-2) ! \cdot 2 !}, \ldots, \frac{\mathbf{u}^{(0, j)}}{j !}\right)
$$

and let further $U: \mathbb{R}^{2} \rightarrow \mathbb{R}^{(k+1)(k+2) / 2}$ be

$$
U(\mathbf{u}):=\left(1, U_{1}(\mathbf{u}), U_{2}(\mathbf{u}), \ldots, U_{k}(\mathbf{u})\right)^{T}
$$

Moreover, for $j=1, \ldots, k$ and $\mathbf{h}=\left(h_{1}, h_{2}\right)$ define

$$
\theta(\mathbf{x})=\left(f(\mathbf{x}), \mathbf{h}^{1} f^{(1)}(\mathbf{x}), \mathbf{h}^{2} f^{(2)}(\mathbf{x}), \ldots, \mathbf{h}^{k} f^{(k)}(\mathbf{x})\right)^{T},
$$

where

$$
\mathbf{h}^{j} f^{(j)}:=\left(\mathbf{h}^{(j, 0)} \cdot D^{(j, 0)} f, \mathbf{h}^{(j-1,1)} \cdot D^{(j-1,1)} f, \mathbf{h}^{(j-2,2)} \cdot D^{(j-2,2)} f, \ldots, \mathbf{h}^{(0, j)} \cdot D^{(0, j)} f\right)
$$

with the multi-index notation $\mathbf{h}^{\boldsymbol{\alpha}}$ and $D^{\left(\alpha_{1}, \alpha_{2}\right)}$ as defined in (4) and (2), respectively. Let $K: \mathbb{R}^{2} \rightarrow \mathbb{R}_{0}^{+}$be a kernel function as specified in Assumption 1 below. Recall that, given the above notation, the quantity

$$
\hat{\theta}(\mathbf{x}):=\operatorname{argmin}_{\boldsymbol{\theta} \in \mathbb{R}^{(k+1)(k+2) / 2}} \sum_{i_{1}, i_{2}=1}^{n}\left[Y_{\left(i_{1}, i_{2}\right)}-\theta(\mathbf{x})^{T} U\left(\frac{\mathbf{t}_{\mathbf{i}}-\mathbf{x}}{\mathbf{h}}\right)\right]^{2} K\left(\frac{\mathbf{t}_{\mathbf{i}}-\mathbf{x}}{\mathbf{h}}\right)
$$

is called local polynomial estimator of order $k$ of $\theta(x)$ and that the statistic

$$
\hat{f}_{n}(\mathbf{x})=U^{T}(\mathbf{0}) \hat{\theta}_{n}(\mathbf{x})
$$

is called local polynomial estimator of order $k$ of $f(\mathbf{x})$ (see Tsybakov (2009)). Introducing some more notation we can rewrite the estimators $\hat{\theta}_{n}(\mathbf{x})$ and $\hat{f}_{n}(\mathbf{x})$ in a perhaps more intuitive way. For $\mathbf{x} \in \bar{\Omega}$ let $\mathbf{a}_{n, \mathbf{x}} \in \mathbb{R}^{(k+1)(k+2) / 2}$ and $\mathbf{B}_{n, \mathbf{x}} \in \mathbb{R}^{(k+1)(k+2) / 2 \times(k+1)(k+2) / 2}$ be defined as

$$
\mathbf{a}_{n, \mathbf{x}}:=\frac{1}{n^{2} h_{1} h_{2}} \sum_{i_{1}, i_{2}=1}^{n} Y_{\left(i_{1}, i_{2}\right)} U\left(\frac{\mathbf{t}_{\mathbf{i}}-\mathbf{x}}{\mathbf{h}}\right) K\left(\frac{\mathbf{t}_{\mathbf{i}}-\mathbf{x}}{\mathbf{h}}\right)
$$


and

$$
\mathrm{B}_{n, \mathbf{x}}:=\frac{1}{n^{2} h_{1} h_{2}} \sum_{i_{1}, i_{2}=1}^{n} U\left(\frac{\mathbf{t}_{\mathbf{i}}-\mathbf{x}}{\mathbf{h}}\right) U^{T}\left(\frac{\mathbf{t}_{\mathbf{i}}-\mathbf{x}}{\mathbf{h}}\right) K\left(\frac{\mathbf{t}_{\mathbf{i}}-\mathbf{x}}{\mathbf{h}}\right) .
$$

Now we can write

$$
\hat{\theta}_{n}(\mathbf{x})=\operatorname{argmin}_{\boldsymbol{\theta} \in \mathbb{R}^{(k+1)(k+2) / 2}}\left(-2 \boldsymbol{\theta}^{T} \mathbf{a}_{n, \mathbf{x}}+\boldsymbol{\theta}^{T} \mathbf{B}_{n, \mathbf{x}} \theta\right)
$$

which yields the necessary condition

$$
\mathrm{B}_{n, \mathbf{x}} \hat{\theta}_{n}(\mathbf{x})=\mathbf{a}_{n, \mathbf{x}}
$$

It is obvious that for a positive definite matrix $B_{n, \mathbf{x}}$ the estimator $\hat{\theta}_{n}(\mathbf{x})$ is defined by the equation $\hat{\theta}_{n}(\mathbf{x})=\mathbf{B}_{n, \mathbf{x}}^{-1} \mathbf{a}_{n, \mathbf{x}}$ and that, also for a positive definite matrix $\mathbf{B}_{n, \mathbf{x}}$, with the definition of the weights $W_{n, \mathbf{i}}(\mathbf{x})$ by

$$
W_{n, \mathbf{i}}(\mathbf{x})=\frac{1}{n^{2} h_{1} h_{2}} U^{T}(\mathbf{0}) \mathbf{B}_{n, \mathbf{x}}^{-1} U\left(\frac{\mathbf{t}_{\mathbf{i}}-\mathbf{x}}{\mathbf{h}}\right) K\left(\frac{\mathbf{t}_{\mathbf{i}}-\mathbf{x}}{\mathbf{h}}\right)
$$

we obtain

$$
\hat{f}_{n}(\mathbf{x})=\sum_{i_{1}, i_{2}=1}^{n} Y_{\left(i_{1}, i_{2}\right)} W_{n, \mathbf{i}}(\mathbf{x})
$$

i.e., the estimator $\hat{f}_{n}(\mathbf{x})$ is linear in $Y_{\mathbf{i}}$.

Lemma 1. Let $\boldsymbol{B}_{n, \mathbf{x}}$ be as defined in (6), $K$ a kernel as specified in Assumption 1 and define the matrices $\mathbf{B}, \mathbf{B}_{\mathbf{x}} \in \mathbb{R}^{(k+1)(k+2) / 2 \times(k+1)(k+2) / 2}$ as

$$
\mathbf{B}:=\int_{\mathbb{R}^{2}} U(\mathbf{u}) U^{T}(\mathbf{u}) K(\mathbf{u}) d \mathbf{u}, \quad \text { and } \quad \mathbf{B}_{\mathbf{x}}:=g_{1}\left(x_{1}\right) g_{2}\left(x_{2}\right) \cdot \mathbf{B}
$$

where integration is carried out component-wise. Let further Assumption 2 be satisfied. Then 
(i) for each $(p, q) \in\{1, \ldots,(k+1)(k+2) / 2\} \times\{1, \ldots,(k+1)(k+2) / 2\}, 0<\delta<1 / 2$,

$$
\sup _{\mathbf{x} \in[\delta, 1-\delta]^{2}}\left|\mathbf{B}_{n, \mathbf{x}}-\mathbf{B}_{\mathbf{x}}\right|_{p, q}=O\left(h_{1}+h_{2}\right)
$$

and

(ii) the matrix $\mathrm{B}_{\mathbf{x}}$ is positive definite.

Note that the matrix $\mathbf{B}$ is independent of the variable $\mathbf{x}$.

Lemma 2. Let $\mathrm{B}$ be as defined in (8), $K$ a kernel as specified in Assumption 1 and define

$$
\widetilde{K}_{\mathbf{B}, U}(\mathbf{u}):=U^{T}(\mathbf{0}) \boldsymbol{B}^{-1} U(\mathbf{u}) K(\mathbf{u}) \text { and } s(\mathbf{x}):=\sigma\left\|\widetilde{K}_{\mathcal{B}, U}\right\|_{2} \frac{1}{\sqrt{g_{1}\left(x_{1}\right) g_{2}\left(x_{2}\right)}}
$$

then

$$
\operatorname{Var}\left[\hat{f}_{n}(\mathbf{x})\right]=\frac{s^{2}(\mathbf{x})}{n^{2} h_{1} h_{2}}+o\left(\frac{1}{n^{2} h_{1} h_{2}}\right)
$$

where the estimate $o\left(\frac{1}{n^{2} h_{1} h_{2}}\right)$ is independent of the variable $\mathbf{x}$.

\subsection{A limit theorem and its implications}

Given the notation and the auxiliary results presented in the previous Section 3.1 we can now state the main results for the two-dimensional regression model (1).

Theorem 1. Assume that $\mathbb{E}\left|\varepsilon_{(1,1)}\right|^{r}<\infty$ for some $r>4 /(2-\delta), \delta \in(0,1]$. Let Assumption 1 and Assumption 2 be satisfied and assume $\sqrt{\log (n)}\left(1 / n^{\delta} h_{1} h_{2}+1 / n h_{1}^{2}+1 / n h_{2}^{2}\right)=o(1)$. Further assume that there exist constants $0<l<1$ and $L<\infty$ such that the inequality $h_{1}+h_{2} \leq L\left(h_{1} h_{2}\right)^{1-l}$ holds. Then, for all $0<\delta<1 / 2, \kappa \in \mathbb{R}$

$$
\begin{gathered}
\lim _{n \rightarrow \infty} \mathbb{P}\left(\sup _{\mathbf{x} \in[\delta, 1-\delta]^{2}}\left(s(\mathbf{x})^{-1} n \sqrt{h_{1} h_{2}}\left|\hat{f}_{n}(\mathbf{x})-\mathbb{E} \hat{f}_{n}(\mathbf{x})\right|-l_{n}\right) l_{n}<\kappa\right)=e^{-2 e^{-\kappa}} \\
l_{n}:=\sqrt{2 \log \left(C_{2} /\left(h_{1} h_{2}\right)\right)}+\frac{\log \left(2 \log \left(C_{2} /\left(h_{1} h_{2}\right)\right)\right)}{2 \sqrt{2 \log \left(C_{2} /\left(h_{1} h_{2}\right)\right)}}
\end{gathered}
$$


and

$$
\begin{aligned}
C_{2}=\frac{(1-2 \delta)^{2} \cdot(2 \pi)^{-3 / 2}}{\left\|\widetilde{K}_{\mathrm{B}, U}\right\|^{2}}\left(\int_{\mathbb{R}^{2}} \widetilde{K}_{\mathrm{B}, U}(\mathbf{u}) D^{(2,0)} \widetilde{K}_{\mathfrak{B}, U}(\mathbf{u}) d \mathbf{u} \int_{\mathbb{R}^{2}} \widetilde{K}_{\mathrm{B}, U}(\mathbf{u}) D^{(0,2)} \widetilde{K}_{\mathcal{B}, U}(\mathbf{u}) d \mathbf{u}\right. \\
\left.-\left(\int_{\mathbb{R}^{2}} \widetilde{K}_{\mathrm{B}, U}(\mathbf{u}) D^{(1,1)} \widetilde{K}_{\mathfrak{B}, U}(\mathbf{u}) d \mathbf{u}\right)^{2}\right)^{\frac{1}{2}} .
\end{aligned}
$$

It is clear that in nonparametric curve estimation one always has to deal with the effect of bias subject to smoothing. In the context of the construction of (simultaneous) confidence bands one of two major strategies to cope with this difficulty is usually pursued, namely explicit bias correction, which allows for an "optimal" choice of smoothing parameter and slight undersmoothing, i.e., accepting a higher variability in the estimation in order to suppress the bias. In this paper we shall follow the latter strategy for which Hall (1992) gave theoretical justification by showing that it results in minimal coverage error as compared to explicit bias correction. The price, however, are slightly wider asymptotic bands. As a direct consequence of Theorem 1 and the use of an undersmoothing bandwidth we obtain the following result.

Corollary 1. Let the assumptions of Theorem 1 be satisfied and let $\left(h_{1}+h_{2}\right)^{k+a} \cdot n \cdot \sqrt{h_{1} h_{2} \log (n)}=o(1)$. Then the set

$$
\left\{\left[\hat{f}_{n}(\mathbf{x})-\Phi_{n, \alpha}(\mathbf{x}), \hat{f}_{n}(\mathbf{x})-\Phi_{n, \alpha}(\mathbf{x})\right] \mid \mathbf{x} \in[\delta, 1-\delta]^{2}\right\}
$$

where

$$
\Phi_{n, \alpha}(\mathbf{x}):=\left(\frac{\kappa_{\alpha}}{l_{n}}+l_{n}\right) \frac{s(\mathbf{x})}{n \sqrt{h_{1} h_{2}}} \quad \text { and } \quad \kappa_{\alpha}=-\log (-0.5 \log (1-\alpha))
$$

defines an asymptotic uniform $(1-\alpha)$-confidence band for the bivariate function $f \in C^{k, a}(\bar{\Omega})$ from regression model (1).

\section{Multivariate nonparametric regression}

In this section we first introduce more notation that is needed in order to define the local polynomial estimator of order $k$ for the multivariate function $f$. Then we state the $d$-dimensional versions of Theorem 1 and Corollary 1 presented in the previous section and conclude with a further generalization regarding 
the estimator. For $j=1, \ldots, k$ and $N_{j, d}:=\left(\begin{array}{c}d+j \\ d\end{array}\right)$ let

$$
\Psi_{j}:\left\{1, \ldots, N_{j, d-1}\right\} \rightarrow\left\{\boldsymbol{\alpha} \in\{0,1, \ldots, j\}^{d}|| \boldsymbol{\alpha} \mid \leq j\right\}
$$

be an enumeration of the set $\left\{\boldsymbol{\alpha} \in\{0,1, \ldots, j\}^{d}|| \boldsymbol{\alpha} \mid \leq j\right\}$ and let $U: \mathbb{R}^{d} \rightarrow \mathbb{R}^{N_{k, d}}$ be defined as

$$
U(\mathbf{u}):=\left(1, U_{1, \Psi_{1}(1)}(\mathbf{u}), \ldots, U_{1, \Psi_{1}(d)}(\mathbf{u}), \ldots, U_{k, \Psi_{k}(1)}(\mathbf{u}), \ldots, U_{k, \Psi_{k}\left(N_{k, d-1}\right)}(\mathbf{u})\right)
$$

where

$$
U_{j, \Psi_{j}(p)}(\mathbf{u})=\frac{\mathbf{u}^{\Psi_{j}(p)}}{\Psi_{j}(p) !}, p=1, \ldots, N_{j, d-1}, j=1, \ldots, k
$$

Moreover, for $j=1, \ldots, k$ and $\mathbf{h}=\left(h_{1}, \ldots, h_{d}\right)$ define

$$
\theta(\mathbf{x})=\left(f(\mathbf{x}), \mathbf{h}^{1} f^{(1)}(\mathbf{x}), \mathbf{h}^{2} f^{(2)}(\mathbf{x}), \ldots, \mathbf{h}^{k} \cdot f^{(k)}(\mathbf{x})\right)^{T}
$$

where

$$
\mathbf{h}^{j} f^{(j)}:=\left(\mathbf{h}^{\Psi_{j}(1)} \cdot D^{\Psi_{j}(1)} f, \ldots, \mathbf{h}^{\Psi_{j}\left(N_{j, d-1}\right)} \cdot D^{\Psi_{j}\left(N_{j, d-1}\right)} f\right) .
$$

Now we can define the $d$-dimensional local polynomial estimator of order $k$ of $f(\mathbf{x})$ by

$$
\hat{f}_{n}(\mathbf{x})=U^{T}(\mathbf{0}) \hat{\theta}_{n}(\mathbf{x})
$$

where

$$
\hat{\theta}(\mathbf{x}):=\operatorname{argmin}_{\boldsymbol{\theta} \in \mathbb{R}^{N_{k, d}}} \sum_{i_{1}, \ldots, i_{d}=1}^{n}\left[Y_{\mathbf{i}}-\theta(\mathbf{x})^{T} U\left(\frac{\mathbf{t}_{\mathbf{i}}-\mathbf{x}}{\mathbf{h}}\right)\right]^{2} K\left(\frac{\mathbf{t}_{\mathbf{i}}-\mathbf{x}}{\mathbf{h}}\right)
$$

and $K: \mathbb{R}^{d} \rightarrow \mathbb{R}_{0}^{+}$is a kernel function as specified in Assumption 1 above. For $\mathbf{x} \in \bar{\Omega}$ let $\mathbf{a}_{n, \mathbf{x}, d} \in \mathbb{R}^{N_{k, d}}$ and $\boldsymbol{B}_{n, \mathbf{x}, d} \in \mathbb{R}^{N_{k, d} \times N_{k, d}}$ be the $d$-dimensional analogues of $\mathbf{a}_{n, \mathbf{x}}$ and $\boldsymbol{B}_{n, \mathbf{x}}$, i.e.,

$$
\mathbf{a}_{n, \mathbf{x}, d}:=\frac{1}{n^{d} h_{1} \cdot \ldots \cdot h_{d}} \sum_{i_{1}, \ldots, i_{d}=1}^{n} Y_{\mathbf{i}} U\left(\frac{\mathbf{t}_{\mathbf{i}}-\mathbf{x}}{\mathbf{h}}\right) K\left(\frac{\mathbf{t}_{\mathbf{i}}-\mathbf{x}}{\mathbf{h}}\right)
$$


and

$$
\mathrm{B}_{n, \mathbf{x}, d}:=\frac{1}{n^{d} h_{1} \cdot \ldots \cdot h_{d}} \sum_{i_{1}, i_{2}=1}^{n} U\left(\frac{\mathbf{t}_{\mathbf{i}}-\mathbf{x}}{\mathbf{h}}\right) U^{T}\left(\frac{\mathbf{t}_{\mathbf{i}}-\mathbf{x}}{\mathbf{h}}\right) K\left(\frac{\mathbf{t}_{\mathbf{i}}-\mathbf{x}}{\mathbf{h}}\right) .
$$

Again we have

$$
\hat{\theta}_{n}(\mathbf{x})=\operatorname{argmin}_{\boldsymbol{\theta} \in \mathbb{R}^{N} k, d}\left(-2 \boldsymbol{\theta}^{T} \mathbf{a}_{n, \mathbf{x}, d}+\boldsymbol{\theta}^{T} \mathbf{B}_{n, \mathbf{x}, d} \theta\right) \text { and } \hat{f}_{n}(\mathbf{x})=\sum_{i_{1}, \ldots, i_{d}=1}^{n} Y_{\mathbf{i}} W_{n, \mathbf{i}, d}(\mathbf{x}),
$$

with weights

$$
W_{n, \mathbf{i}, d}(\mathbf{x})=\frac{1}{n^{d} h_{1} \cdot \ldots \cdot h_{d}} U^{T}(\mathbf{0}) \boldsymbol{B}_{n, \mathbf{x}, d}^{-1} U\left(\frac{\mathbf{t}_{\mathbf{i}}-\mathbf{x}}{\mathbf{h}}\right) K\left(\frac{\mathbf{t}_{\mathbf{i}}-\mathbf{x}}{\mathbf{h}}\right)
$$

provided the matrix $\boldsymbol{B}_{n, \mathbf{x}, d}$ is positive definite.

Theorem 2. Assume that $\mathbb{E}\left|\varepsilon_{(1, \ldots, 1)}\right|^{r}<\infty$ for some $r>2 d /(d-\delta), \delta \in(0,1], \delta \neq d$. Let Assumption 1 and Assumption 2 hold and let $\sqrt{\log (n)}\left(1 /\left(n^{\delta} h_{1} \cdot \ldots \cdot h_{d}\right)+1 /\left(n h_{1}^{d}\right)+\ldots+1 /\left(n h_{d}^{d}\right)\right)=o(1)$. Further assume that there exist constants $0<l<1$ and $L<\infty$ such that the inequality $\sum_{p=1}^{d} h_{p} \leq L\left(\prod_{p=1}^{d} h_{p}\right)^{1-l}$ holds. Then, for all $0<\delta<1 / 2, \kappa \in \mathbb{R}, s_{d}(\mathbf{x}):=\sigma\left\|\widetilde{K}_{\mathrm{B}, U}\right\| / \sqrt{g_{1}\left(x_{1}\right) \cdot \ldots \cdot g_{d}\left(x_{d}\right)}$

$$
\begin{gathered}
\lim _{n \rightarrow \infty} \mathbb{P}\left(\sup _{\mathbf{x} \in[\delta, 1-\delta]^{2}}\left(s_{d}(\mathbf{x})^{-1} n^{\frac{d}{2}} \sqrt{h_{1} \cdot \ldots \cdot h_{d}}\left|\hat{f}_{n}(\mathbf{x})-\mathbb{E} \hat{f}_{n}(\mathbf{x})\right|-l_{n}\right) l_{n}<\kappa\right),=e^{-2 e^{-\kappa}} \\
l_{n}:=\sqrt{2 \log \left(C_{d} /\left(h_{1} \cdot \ldots \cdot h_{d}\right)\right)}+(d-1) \frac{\log \left(2 \log \left(C_{d} /\left(h_{1} \cdot \ldots \cdot h_{d}\right)\right)\right.}{2 \sqrt{2 \log \left(C_{d} /\left(h_{1} \cdot \ldots \cdot h_{d}\right)\right)}} \\
\Lambda_{2}:=\left(\frac{1}{\left\|\widetilde{K}_{\mathfrak{B}, U}\right\|} \int_{\mathbb{R}^{d}} \widetilde{K}_{\mathcal{B}, U}(\mathbf{u}) \frac{\partial^{2}}{\partial u_{i} \partial u_{j}} \widetilde{K}_{\mathbf{B}, U}(\mathbf{u}) d \mathbf{u}\right)_{i, j=1}^{d}
\end{gathered}
$$

and

$$
C_{d}=(1-2 \delta)^{d} \cdot(2 \pi)^{-(d+1) / 2} \sqrt{\operatorname{det}\left(\Lambda_{2}\right)}
$$

Corollary 2. Let the assumptions of Theorem 1 be satisfied and let $\left(h_{1}+\ldots+h_{2}\right)^{k+a} \cdot n^{\frac{d}{2}} \cdot \sqrt{h_{1} \cdot \ldots \cdot h_{d} \log (n)}=$ 
$o(1)$. Then the set

$$
\left\{\left[\hat{f}_{n}(\mathbf{x})-\Phi_{n, \alpha, d}(\mathbf{x}), \hat{f}_{n}(\mathbf{x})-\Phi_{n, \alpha, d}(\mathbf{x})\right] \mid \mathbf{x} \in[\delta, 1-\delta]^{d}\right\}
$$

where $\Phi_{n, \alpha, d}(\mathbf{x}):=\left(\frac{\kappa_{\alpha}}{l_{n}}+l_{n}\right) \frac{s_{d}(\mathbf{x})}{n^{\frac{d}{2}} \sqrt{h_{1} \cdot \ldots \cdot h_{d}}} \quad$ and $\quad \kappa_{\alpha}=-\log (-0.5 \log (1-\alpha))$, defines an asymptotic uniform $(1-\alpha)$-confidence band for the multivariate function $f \in C^{k, a}(\bar{\Omega})$ from regression model (1).

The results stated above hold for general linear nonparametric kernel regression estimates with a kernel of convolution form, or a sequence of kernels even, satisfying Assumption 1. This is a consequence of the fact that they can be approximated by a suitable stationary Gaussian process, where the supremum is taken with respect to a growing system of sets, which has an extreme value limit distribution. To conclude the section we now present another limit theorem in which this generalization is formalized.

Theorem 3. Assume that the conditions of Theorem 2 are satisfied and that

$$
\hat{f}_{n}(\mathbf{x})=\frac{1}{n^{d} h_{1}^{d} \cdot \ldots \cdot h_{d}} \sum_{i_{1}, \ldots, i_{d}=1}^{n} Y_{\mathbf{i}} K^{(n)}\left(\frac{\mathbf{t}_{\mathbf{i}}-\mathbf{x}}{\mathbf{h}}\right)
$$

with a sequence of kernels $\left(K^{(n)}\right)_{n \in \mathbb{N}}$ meeting Assumption 1 and one of the following two conditions

(i) There exists a number $M \in \mathbb{N}$, kernels $K, K_{1}, \ldots, K_{M}$, each satisfying Assumption 1, and sequences $\left(a_{n, 1}\right)_{n \in \mathbb{N}}, \ldots,\left(a_{M, n}\right)_{n \in \mathbb{N}}$ such that $a_{p, n}=o\left(\frac{1}{\sqrt{\log (n)}}\right), p=1, \ldots, M$ and

$$
K^{(n)}-K=\sum_{p=1}^{M} a_{n, p} K_{p}
$$

(ii) There exists a limit kernel $K$, meeting Assumption 1 such that

$$
\frac{\left\|K^{(n)}-K\right\|_{\infty}}{\sqrt{h_{1} \cdot \ldots \cdot h_{d}}}=o(1 / \sqrt{\log (n)})
$$

Then Theorem 2 holds when each $\widetilde{K}_{\mathrm{B}, U}$ is to be replaced by $K$ in the definitions of the quantities $s_{d}(\mathbf{x})$ and $\Lambda_{2}$.

Remark 2. This is relevant in several applications. For instance, in the context of spline smoothing, Silverman (1984) showed that a one dimensional cubic spline estimator is asymptotically of convolution- 
kernel form with a bounded, smooth kernel $K_{S}$ defined by $K_{S}(u)=1 / 2 \exp (-|u| / \sqrt{2}) \sin (|u| / \sqrt{2}+\pi / 4)$. The associated estimator satisfies the assumptions of Theorem 3 except for Assumption 1 (i). The results of this paper also hold in this case, even for relatively mild (polynomial) decay of the kernel function in each direction, however, the technical complexity is unproportionally greater, hence we will not include this case to our considerations.

\section{$5 \quad$ Finite sample properties}

In this section the finite sample properties of the proposed asymptotic confidence bands are investigated. First, the simulation setup is described in section 5.1 and the results are presented and discussed in section 5.2 .
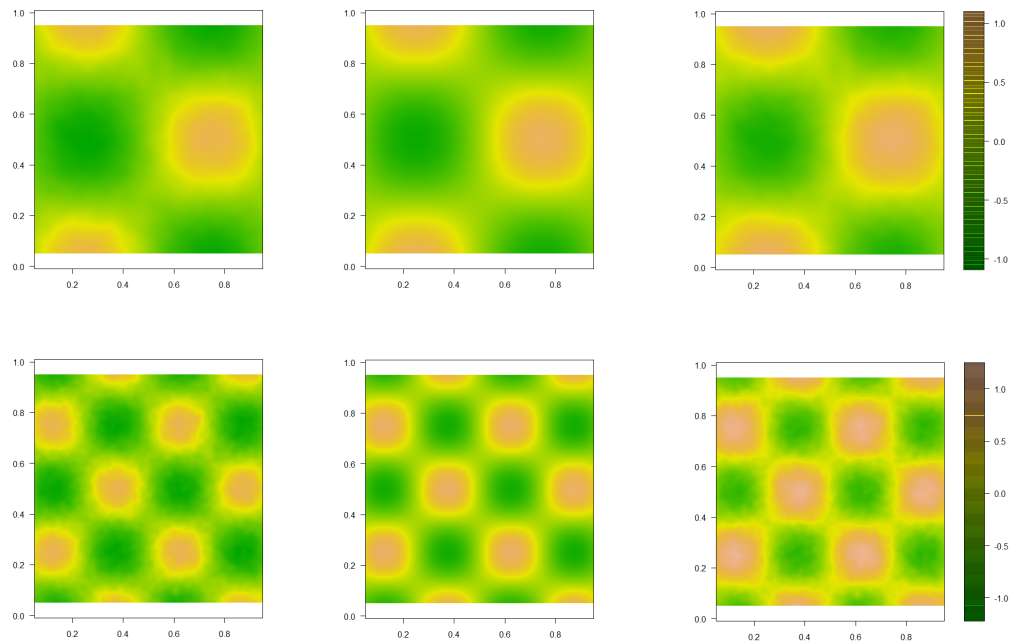

Figure 1: Contour plots of lower confidence surfaces (left), true regression surface (middle) and upper surface (right) based on the local linear estimator and $n=250$ for $f_{1}$ (upper panel) and $f_{2}$ (lower panel).

\subsection{Simulation setup}

All results are based on 2500 simulation runs. We simulate data from the bivariate regression model (1) with normally distributed errors $\varepsilon_{i, j} \sim \mathcal{N}\left(0, \sigma^{2}\right)$ where $\sigma=0.3$ and $\left(n_{1}, n_{2}\right) \in\{1, \ldots, n\}^{2}, n \in$ $\{75,150,250\}$. For the unknown regression function we consider two different versions $f_{1}$ and $f_{2}$ of a 
product of trigonometric functions defined by

$$
f_{j}\left(x_{1}, x_{2}\right)=-\sin \left(2 j \pi\left(x_{1}-0.5\right)\right) \cos \left(2 j \pi x_{2}\right), j=1,2
$$

with increasing complexity (see Figure 1, central column for a contour plot of both functions under consideration). As kernel function $K$ we consider a product kernel $K\left(x_{1}, x_{2}\right)=K_{1}\left(x_{1}\right) \cdot K_{1}\left(x_{2}\right)$ with a compactly supported, three times continuously differentiable function $K_{1}(x)=\left(1-x^{2}\right)^{4} I_{[-1,1]}(x)$. In these settings we compare the performances of both a local linear as well as a local quadratic estimator. The corresponding limit kernels $\widetilde{K}_{\mathrm{B}, U \text {,lin }}$ and $\widetilde{K}_{\mathrm{B}, U \text {,quad }}$ are then given by $\widetilde{K}_{\mathrm{B}, U, \text { lin }}(\mathbf{x})=1.514 \cdot K(\mathbf{x})$ and $\widetilde{K}_{\mathbf{B}, U, \text { quad }}(\mathbf{x})=\left(3.482-10.826\left(x_{1}^{2}+x_{2}^{2}\right)\right) K(\mathbf{x})$. A difference based variance estimator is used to estimate $\sigma^{2}$. Concerning the smoothing parameter $h$ we first determine a suitable value for each setting by a small preliminary simulation study. These fixed smoothing parameters are then used in all runs for the respective simulation setting.
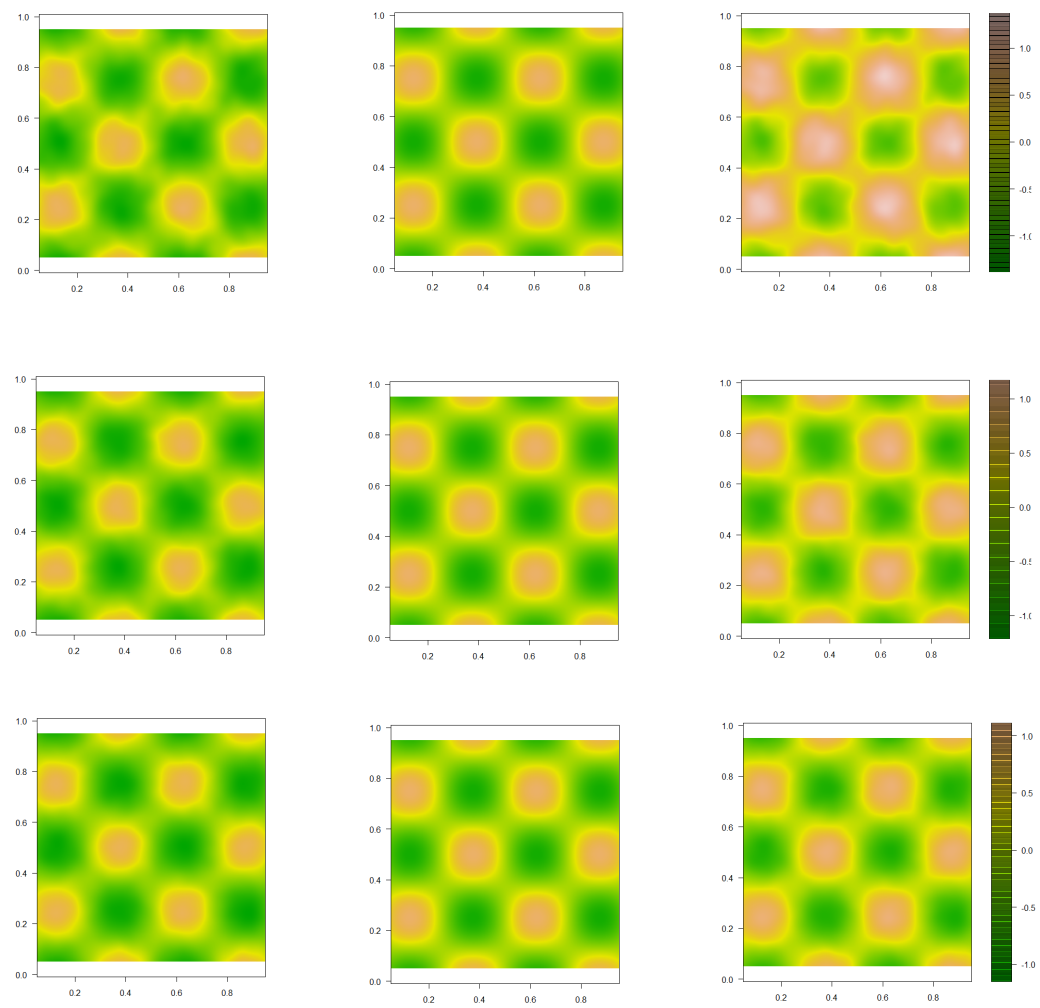

Figure 2: Contour plots of the lower (left) and upper (right) confidence surfaces for the regression function $f_{2}$ based on the local quadratic estimator and each of 75 (upper panel), 150 (middle panel) and 250 (lower panel) observations. The true regression surface is shown in the central column. 


\subsection{Simulation results}

Table 1: Simulated coverage probabilities and mean half-lengths of the confidence bands for the functions $f_{1}$ and $f_{2}$ and the local linear estimator.

\begin{tabular}{|c|c|c|c|c|c|c|c|}
\hline \multirow[b]{2}{*}{$n$} & \multirow[b]{2}{*}{$f$} & \multicolumn{2}{|c|}{$90 \%$ nominal coverage } & \multicolumn{2}{|c|}{$95 \%$ nominal coverage } & \multicolumn{2}{|c|}{$99 \%$ nominal coverage } \\
\hline & & Cov. $(\%)$ & Length & Cov. $(\%)$ & Length & Cov. $(\%)$ & Length \\
\hline \multirow{2}{*}{250} & $f_{1}$ & 85.86 & 0.083 & 93.20 & 0.087 & 99.00 & 0.097 \\
\hline & $f_{2}$ & 88.68 & 0.173 & 95.64 & 0.179 & 99.44 & 0.197 \\
\hline \multirow{2}{*}{150} & $f_{1}$ & 88.92 & 0.133 & 95.04 & 0.140 & 99.08 & 0.148 \\
\hline & $f_{2}$ & 87.92 & 0.225 & 94.04 & 0.234 & 99.28 & 0.258 \\
\hline \multirow{2}{*}{75} & $f_{1}$ & 87.24 & 0.224 & 94.00 & 0.236 & 99.36 & 0.264 \\
\hline & $f_{2}$ & 89.36 & 0.326 & 94.44 & 0.342 & 99.24 & 0.379 \\
\hline
\end{tabular}

Table 2: Simulated coverage probabilities and mean half-lengths of the confidence bands for the functions $f_{1}$ and $f_{2}$ and the local quadratic estimator.

\begin{tabular}{|c|c|c|c|c|c|c|c|}
\hline \multirow[b]{2}{*}{$n$} & \multirow[b]{2}{*}{$f$} & \multicolumn{2}{|c|}{$90 \%$ nominal coverage } & \multicolumn{2}{|c|}{$95 \%$ nominal coverage } & \multicolumn{2}{|c|}{$99 \%$ nominal coverage } \\
\hline & & Cov. $(\%)$ & Length & Cov. $(\%)$ & Length & Cov. (\%) & Length \\
\hline \multirow{2}{*}{250} & $f_{1}$ & 88.44 & 0.099 & 94.96 & 0.104 & 99.44 & 0.115 \\
\hline & $f_{2}$ & 89.92 & 0.098 & 94.64 & 0.102 & 98.84 & 0.114 \\
\hline \multirow{2}{*}{150} & $f_{1}$ & 90.40 & 0.145 & 95.68 & 0.152 & 99.16 & 0.168 \\
\hline & $f_{2}$ & 91.80 & 0.143 & 96.24 & 0.149 & 99.60 & 0.166 \\
\hline \multirow{2}{*}{75} & $f_{1}$ & 92.44 & 0.277 & 97.24 & 0.291 & 99.72 & 0.323 \\
\hline & $f_{2}$ & 92.40 & 0.259 & 96.80 & 0.273 & 99.68 & 0.302 \\
\hline
\end{tabular}

We now summarize the results of the simulation study. Figure 2 illustrates the confidence bands based on the local quadratic estimator for the regression function $f_{2}$, top down for growing sample sizes. In each row, the contourplots show the lower confidence surface, the true object and the upper confidence surface (from left to right) and the improvement in the performance for growing $n$ clearly shows. Tables 1 and 2 contain the simulated coverage probabilities and the average half widths of the bands for the local linear and the local quadratic estimator, respectively. We observe that, even for moderate sample sizes, the simulated coverage probabilities are close to the nominal values and that the bands are reasonably narrow. Further it is evident that the bands for $f_{1}$ constructed with the local linear estimator are narrower than the ones for the local quadratic estimator. This is due to the fact that the local linear estimator produces a smaller variance because the $L^{2}$-norms of the limit kernels $\widetilde{K}_{\mathbf{B}, U \text {,lin }}$ and $\widetilde{K}_{\mathrm{B}, U \text {,quad }}$ are not equal, more precisely $\left\|\widetilde{K}_{\mathrm{B}, U, \text { lin }}\right\|_{2}<\left\|\widetilde{K}_{\mathrm{B}, U \text {,quad }}\right\|$. Nevertheless the results for the local quadratic estimator 
are slightly better which is due to the smaller bias of this estimator as compared to its linear counterpart which guarantees a more accurate centering of the bands and results in higher coverage. We also find that, while there seems to be hardly any difference for the different settings for the local quadratic estimator, the bands for $f_{2}$ based on the local linear estimator are clearly wider. The effect is shown in Figure 2 where plots of both cases for the local linear estimator are displayed.

Acknowledgments: This work has been supported by the Collaborative Research Center "Statistical modeling of nonlinear dynamic processes" (SFB 823, Teilprojekt C4) of the German Research Foundation (DFG). The author would like to thank Holger Dette for helpful discussions and a careful reading of this manuscript.

\section{References}

Bickel, P. and Rosenblatt, M. (1973a). Two-dimensional random fields. In Multivariate Analysis, III (Proc. Third Internat. Sympos., Wright State Univ., Dayton, Ohio, 1972), pages 3-15. Academic Press, New York.

Bickel, P. J. and Rosenblatt, M. (1973b). On some global measures of the deviations of density function estimates. Ann. Statist., 1:1071-1095.

Bissantz, N., Dümbgen, L., Holzmann, H., and Munk, A. (2007). Nonparametric confidence bands in deconvolution density estimation. J. R. Stat. Soc. Ser. B Stat. Methodol., 69:483-506.

Claeskens, G. and van Keilegom, I. (2003). Bootstrap confidence bands for regression curves and their derivatives. Ann. Statist., 31:1852-1884.

Dette, H. and Munk, A. (1998). Validation of linear regression models. Ann. Statist., 26(2):778-800.

Eubank, R. L. and Speckman, P. L. (1993). Confidence bands in nonparametric regression. J. Amer. Statist. Assoc., 88:1287-1301.

Giné, E., Koltchinskii, V., and Sakhanenko, L. (2004). Kernel density estimators: convergence in distribution for weighted sup-norms. Probab. Theory Related Fields, 130(2):167-198.

Giné, E. and Nickl, R. (2010). Confidence bands in density estimation. Ann. Statist., 38:1122-1170.

Hall, P. (1992). Effect of bias estimation on coverage accuracy of bootstrap confidence intervals for a probability density. Ann. Statist., 20:675-694.

Hall, P. and Horowitz, J. (2013). A simple bootstrap method for constructing confidence bands for functions. Ann. Statist., 41(4):1892-1921.

Härdle, W. (1989). Asymptotic maximal deviation of m-smoothers. J. Multivariate Anal., pages 163-179.

Härdle, W. and Song, S. (2010). Confidence bands in quantile regression. Econometric Theory, 26(4):11801200 .

Johnston, G. J. (1982). Probabilities of maximal deviations for nonparametric regression function estimates. J. Multivariate Anal., pages 402-414. 
Khoshnevisan, D. (2002). Multiparameter Processes - An Introduction. Springer, New York.

Konakov, V. D. and Piterbarg, V. I. (1984). On the convergence rate of maximal deviation distribution for kernel regression estimate. J. Multivariate Anal., 15:279-294.

Lounici, K. and Nickl, R. (2011). Global uniform risk bounds for wavelet deconvolution estimators. Ann. Statist., 39:201-231.

Neumann, M. H. and Polzehl, J. (1998). Simultaneous bootstrap confidence bands in nonparametric regression. J. Nonparametr. Stat., 9:307-333.

Owen, A. B. (2005). Multidimensional variation for quasi-Monte Carlo. In Contemporary Multivariate Analysis and Design of Experiments, volume 2 of Ser. Biostat., pages 49-74. World Sci. Publ., Hackensack, NJ.

Piterbarg, V. I. (1996). Asymptotic methods in the theory of Gaussian processes and fields, volume 148 of Translations of Mathematical Monographs. American Mathematical Society, Providence, RI.

Rio, E. (1993). Strong approximation for set-indexed partial sum processes via KMT constructions I. Ann. Probab., 21(2):759-790.

Rio, E. (1994). Local invariance principles and their application to density estimation. Probab. Theory Related Fields, 98(1):21-45.

Rosenblatt, M. (1976). On the maximal deviation of k-dimensional density estimates. Ann. Probab., 6:1009-1015.

Sacks, J. and Ylvisaker, D. (1970). Designs for regression problems with correlated errors. Ann. Math. Statist., 41:2057-2074.

Silverman, B. W. (1984). Spline smoothing: the equivalent variable kernel method. Ann. Statist., 12:898916.

Smirnov, N. V. (1950). On the construction of confidence regions for the density of distribution of random variables. Dokl. Akad. Nauk, 74:189-191.

Stadtmüller, U. (1986). Asymptotic properties of nonparametric curve estimates. Period. Math. Hungar., $17: 83-108$.

Tsybakov, A. B. (2009). Introduction to nonparametric estimation. Springer Series in Statistics. Springer, New York.

Wand, M. P. and Jones, M. C. (1995). Kernel smoothing. Monographs on Statistics and Applied Probability, 60. Chapman and Hall, Ltd., London.

Xia, Y. (1998). Bias-corrected confidence bands in nonparametric regression. J. R. Stat. Soc. Ser. B Stat. Methodol., 60:797-811. 


\section{Proofs}

In this section we present the proofs of the results presented in the previous sections. Those that are completely analogous to the ones presented in Tsybakov (2009), Chapter 1.6, are omitted, only some extensions are included in this section.

\subsection{Proofs of auxiliary results}

\section{Proof of Lemma 1}

Since each entry $U^{\left(p_{0}\right)}(\mathbf{u}) U^{\left(q_{0}\right)}(\mathbf{u})$ of the matrix

$$
U(\mathbf{u}) U^{T}(\mathbf{u})=\left(U^{(p)}(\mathbf{u}) U^{q}(\mathbf{u})\right)_{p, q=1}^{(k+1)(k+2) / 2}
$$

is a polynomial (of degree $\leq k$ ) the smoothness properties of $K$ transfer to the products

$$
U^{\left(p_{0}\right)}(\mathbf{u}) U^{\left(q_{0}\right)}(\mathbf{u}) \cdot K
$$

hence it follows by Assumption 2 that

$$
\begin{aligned}
\left(\boldsymbol{B}_{n, \mathbf{x}}\right)_{\left(p_{0}, q_{0}\right)} & =\frac{1}{n^{2} h_{1} h_{2}} \sum_{i_{1}, i_{2}=1}^{n} U^{\left(p_{0}\right)}\left(\frac{\mathbf{t}_{\mathbf{i}}-\mathbf{x}}{\mathbf{h}}\right) U^{\left(q_{0}\right)}\left(\frac{\mathbf{t}_{\mathbf{i}}-\mathbf{x}}{\mathbf{h}}\right) K\left(\frac{\mathbf{t}_{\mathbf{i}}-\mathbf{x}}{\mathbf{h}}\right) \\
& =\frac{1}{h_{1} h_{2}} \int_{0}^{1} \int_{0}^{1} U^{\left(p_{0}\right)}\left(\frac{\mathbf{z}-\mathbf{x}}{\mathbf{h}}\right) U^{\left(q_{0}\right)}\left(\frac{\mathbf{z}-\mathbf{x}}{\mathbf{h}}\right) K\left(\frac{\mathbf{z}-\mathbf{x}}{\mathbf{h}}\right) g_{1}\left(z_{1}\right) g_{2}\left(z_{2}\right) d \mathbf{z}+O\left(\frac{1}{n}\right) \\
& =\int_{-\frac{x_{2}}{h_{2}}}^{\frac{1-x_{2}}{h_{2}}} \int_{-\frac{x_{1}}{h_{1}}}^{\frac{1-x_{1}}{h_{1}}} U^{\left(p_{0}\right)}(\mathbf{u}) U^{\left(q_{0}\right)}(\mathbf{u}) K(\mathbf{u}) g_{1}\left(x_{1}+h_{1} u_{1}\right) g_{2}\left(x_{2}+h_{2} u_{2}\right) d \mathbf{u .}+O\left(\frac{1}{n}\right) .
\end{aligned}
$$

Finally, again by Assumption 2

$$
\left(\mathbf{B}_{n, \mathbf{x}}\right)_{\left(p_{0}, q_{0}\right)}=g_{1}\left(x_{1}\right) g_{2}\left(x_{2}\right) \int_{-1}^{1} \int_{-1}^{1} U^{\left(p_{0}\right)}(\mathbf{u}) U^{\left(q_{0}\right)}(\mathbf{u}) K(\mathbf{u}) d \mathbf{u}+O\left(\frac{1}{n}+h_{1}+h_{2}\right)
$$

for sufficiently large $n$. 


\section{Proof of Lemma 2}

Lemma 1 implies

$$
\begin{aligned}
\operatorname{Var} \hat{f}_{n}(\mathbf{x}) & =\sigma^{2} \sum_{i_{1}, i_{2}=1}^{n}\left(W_{n,\left(i_{1}, i_{2}\right)}(\mathbf{x})\right)^{2} \\
& =\frac{\sigma^{2}}{g_{1}^{2}\left(x_{1}\right) g_{2}^{2}\left(x_{2}\right) n^{4} h_{1}^{2} h_{2}^{2}}\left(\sum_{i_{1}, i_{2}=1}^{n}\left(U^{T}(0) \mathbf{B}^{-1} U\left(\frac{\mathbf{t}_{\mathbf{i}}-\mathbf{x}}{\mathbf{h}}\right) K\left(\frac{\mathbf{t}_{\mathbf{i}}-\mathbf{x}}{\mathbf{h}}\right)\right)^{2}+o(1)\right) .
\end{aligned}
$$

By Assumption 2 and Assumption 1 we obtain

$$
\begin{aligned}
\operatorname{Var} \hat{f}_{n}(\mathbf{x}) & =\frac{\sigma^{2}}{g_{1}^{2}\left(x_{1}\right) g_{2}^{2}\left(x_{2}\right) n^{2} h_{1}^{2} h_{2}^{2}} \sum_{i_{1}, i_{2}=1}^{n}\left(\widetilde{K}_{\mathbf{B}, U}\left(\frac{\mathbf{t}_{\mathbf{i}}-\mathbf{x}}{\mathbf{h}}\right)\right)^{2} g_{1}\left(t_{i_{1}}\right) g_{2}\left(t_{i_{2}}\right)\left(t_{i_{1}}-t_{i_{1}-1}\right)\left(t_{i_{2}}-t_{i_{2}-1}\right) \\
& +o\left(\frac{1}{n^{2} h_{1} h_{2}}\right)
\end{aligned}
$$

and the assertion of the lemma immediately follows.

\subsection{Proofs of Theorem 1 and Corollary 1}

In order to prove Theorem 1 we perform several steps to approximate the quantity $n \sqrt{h_{1} h_{2}} s(\mathbf{x})^{-1}\left(\hat{f}_{n}(\mathbf{x})-\right.$ $\left.\mathbb{E} \hat{f}_{n}(\mathbf{x})\right)$ uniformly in $\mathbf{x} \in[\delta, 1-\delta]^{2}$ by a stationary Gaussian field $Z(\mathbf{x}):=\int_{\mathbb{R}^{2}} \widetilde{K}_{\mathcal{B}, U}(\mathbf{t}-\mathbf{x}) d \mathbf{t}$, where the

function $\widetilde{K}_{\mathrm{B}, U}$ is defined in (9) and the supremum is then taken over the set $\frac{1}{h_{1}} I_{1} \times \frac{1}{h_{2}} I_{2}$. Then we apply Theorem 14.1 in Piterbarg (1996) to this stationary field which will complete the proof of the theorem. Define the process

$$
Z_{n, 0}(\mathbf{x}):=\frac{\sqrt{g_{1}\left(x_{1}\right) g_{2}\left(x_{2}\right)}}{\sigma\left\|\widetilde{K}_{\mathbf{B}, U}\right\| n \sqrt{h_{1} h_{2}}} \sum_{i_{1}, i_{2}=1}^{n} W_{n, \mathbf{i}}(\mathbf{x}) \varepsilon_{\left(i_{1}, i_{2}\right)}
$$


$Z_{n, 0}$ can be decomposed as follows (see Lemma 7 below for details)

$$
\begin{aligned}
Z_{n, 0}(\mathbf{x}) & =\frac{\sqrt{g_{1}\left(x_{1}\right) g_{2}\left(x_{2}\right)}}{\sigma\left\|\widetilde{K}_{\mathbf{B}, U}\right\| n \sqrt{h_{1} h_{2}}} \sum_{i_{1}, i_{2}=1}^{n} \widetilde{K}_{\mathbf{B}_{x}, U}\left(\frac{\mathbf{t}_{\mathbf{i}}-\mathbf{x}}{\mathbf{h}}\right) \varepsilon_{\left(i_{1}, i_{2}\right)}+\frac{\sqrt{g_{1}\left(x_{1}\right) g_{2}\left(x_{2}\right)}}{\sigma\left\|\widetilde{K}_{\mathbf{B}, U}\right\| n \sqrt{h_{1} h_{2}}} \sum_{i_{1}, i_{2}=1}^{n} \widetilde{K}_{\mathbf{R}_{n, x}, U}\left(\frac{\mathbf{t}_{\mathbf{i}}-\mathbf{x}}{\mathbf{h}}\right) \varepsilon_{\left(i_{1}, i_{2}\right)} \\
& =\frac{1}{\sigma\left\|\widetilde{K}_{\mathbf{B}, U}\right\| n \sqrt{h_{1} h_{2} g_{1}\left(x_{1}\right) g_{2}\left(x_{2}\right)}} \sum_{i_{1}, i_{2}=1}^{n} \widetilde{K}_{\mathbf{B}, U}\left(\frac{\mathbf{t}_{\mathbf{i}}-\mathbf{x}}{\mathbf{h}}\right) \varepsilon_{\left(i_{1}, i_{2}\right)} \\
& +\frac{\sqrt{g_{1}\left(x_{1}\right) g_{2}\left(x_{2}\right)}}{\sigma\left\|\widetilde{K}_{\mathbf{B}, U}\right\| n \sqrt{h_{1} h_{2}}} \sum_{i_{1}, i_{2}=1}^{n} \widetilde{K}_{\mathbf{R}_{n, x}, U}\left(\frac{\mathbf{t}_{\mathbf{i}}-\mathbf{x}}{\mathbf{h}}\right) \varepsilon_{\left(i_{1}, i_{2}\right)}=: Z_{n, 1}(\mathbf{x})+R_{n, 0}(\mathbf{x}),
\end{aligned}
$$

where the processes $Z_{n, 1}(\mathbf{x})$ and $R_{n, \mathbf{x}}(\mathbf{x})$ are defined in an obvious manner. In a first approximation step $Z_{n, 0}$ is approximated by $Z_{n, 1}$. In a next step the observation errors are replaced by their partial sums which allows to replace $Z_{n, 1}$ by $Z_{n, 2}$ :

$$
Z_{n, 2}(\mathbf{x}):=\frac{1}{\left\|\widetilde{K}_{\mathrm{B}, U}\right\| n \sqrt{\prod_{j=1}^{2} g_{j}\left(x_{j}\right) h_{j}}} \sum_{i_{1}, i_{2}=1}^{n} \Delta\left(\mathbf{z} \mapsto \widetilde{K}_{\mathrm{B}, U}\left(\frac{G^{-1}(\mathbf{z})-\mathbf{x}}{\mathbf{h}}\right) ;\left[\frac{\mathbf{i}-\mathbf{1}}{n+1}, \frac{\mathbf{i}}{n+1}\right]\right) W\left(i_{1}, i_{2}\right),
$$

where $W$ is the Wiener sheet specified in Lemma 3,

$$
\begin{aligned}
& \Delta\left(\mathbf{z} \mapsto \widetilde{K}_{\mathrm{B}, U}\left(\frac{G^{-1}(\mathbf{z})-\mathbf{x}}{\mathbf{h}}\right) ;\left[\frac{\mathbf{i}-\mathbf{1}}{n+1}, \frac{\mathbf{i}}{n+1}\right]\right) \\
& \quad=\widetilde{K}_{\mathrm{B}, U}\left(\frac{\mathbf{t}_{\left(i_{1}, i_{2}\right)}-\mathbf{x}}{\mathbf{h}}\right)-\widetilde{K}_{\mathrm{B}, U}\left(\frac{\mathbf{t}_{\left(i_{1}-1, i_{2}\right)}-\mathbf{x}}{\mathbf{h}}\right)-\widetilde{K}_{\mathrm{B}, U}\left(\frac{\mathbf{t}_{\left(i_{1}, i_{2}-1\right)}-\mathbf{x}}{\mathbf{h}}\right)+\widetilde{K}_{\mathrm{B}, U}\left(\frac{\mathbf{t}_{\left(i_{1}-1, i_{2}-1\right)}-\mathbf{x}}{\mathbf{h}}\right)
\end{aligned}
$$

and $G^{-1}(\mathbf{z}):=\left(G_{1}^{-1}\left(z_{1}\right), G_{2}^{-1}\left(z_{2}\right)\right)$. To this end we extend an approach introduced by Stadtmüller (1986) or Eubank and Speckman (1993) for one-dimensional models with deterministic (close to) uniform design. Note that it is not immediate how to generalize this methodology to higher dimensions as well as a not necessarily uniform design under general design assumptions and a broader class of estimators, which is all here. Next, the sum is approximated by the corresponding Wiener integral which gives the approximation of $Z_{n, 2}$ by $Z_{n, 3}$, defined by

$$
Z_{n, 3}(\mathbf{x}):=\frac{1}{\left\|\widetilde{K}_{\mathrm{B}, U}\right\| \sqrt{\prod_{j=1}^{2} g_{j}\left(x_{j}\right) h_{j}}} \int_{I} \widetilde{K}_{\mathrm{B}, U}\left(\frac{\mathbf{z}-\mathbf{x}}{\mathbf{h}}\right) d W(G(\mathbf{z}))
$$


where $G(\mathbf{z}):=\left(G_{1}\left(z_{1}\right), G_{2}\left(z_{2}\right)\right)$. We now define

$$
Z_{n, 4}(\mathbf{x}):=\frac{1}{\left\|\widetilde{K}_{\mathrm{B}, U}\right\| \sqrt{\prod_{j=1}^{2} g_{j}\left(x_{j}\right) h_{j}}} \int_{I} \widetilde{K}_{\mathbf{B}, U}\left(\frac{\mathbf{z}-\mathbf{x}}{\mathbf{h}}\right) \sqrt{g_{1}\left(z_{1}\right) g_{2}\left(z_{2}\right)} d W(\mathbf{z}) .
$$

Note that $Z_{n, 3}$ and $Z_{n_{4}}$ have the same probability structure, i.e., $\left\{Z_{n, 3}(\mathbf{x})\right\} \stackrel{\mathcal{D}}{=}\left\{Z_{n, 4}(\mathbf{x})\right\}$. Hence, in a next step we replace $Z_{n, 4}$ by $Z_{n, 5}$, defined by

$$
Z_{n, 5}(\mathbf{x}):=\frac{1}{\left\|\widetilde{K}_{\mathfrak{B}, U}\right\| \sqrt{h_{1} h_{2}}} \int_{I} \widetilde{K}_{\mathcal{B}, U}\left(\frac{\mathbf{z}-\mathbf{x}}{\mathbf{h}}\right) d W(\mathbf{z})
$$

In a further step we replace the process $Z_{n, 5}$ by the stationary Process $Z_{n, 6}$

$$
Z_{n, 6}(\mathbf{x}):=\frac{1}{\left\|\widetilde{K}_{\mathcal{B}, U}\right\|} \int_{\mathbb{R}^{2}} \widetilde{K}_{\mathcal{B}, U}(\mathbf{z}-\mathbf{x}) d W(\mathbf{z})
$$

and take the supremum with respect to $\mathbf{x} \in 1 / h_{1}[\delta, 1-\delta] \times 1 / h_{2}[\delta, 1-\delta]$. Last we show that the remainder process $R_{n, 0}$ is negligible, that is $\sup _{\mathbf{x} \in[\delta, 1-\delta]^{2}}\left|R_{n, 0}(\mathbf{x})\right|=o_{P}\left(\log (n)^{-1 / 2}\right)$.

Each approximation step corresponds to one of the lemmas 3 to 7 listed and proven below.

Lemma 3. There exists a Wiener sheet $W$ on a suitable probability space such that

$$
\sup _{x \in I}\left|Z_{n, 1}(x)-Z_{n, 2}(x)\right|=O\left(\frac{\log (n)}{n^{\delta} \sqrt{h_{1} h_{2}}}\right)
$$

where $\delta \in(0,1]$ is the constant defined in Theorem 1 .

Proof. Here and in what follows let $I$ denote the unit cube, i.e., $I:=[0,1]^{d}$. Define the partial sums $S_{\left(i_{1}, i_{2}\right)}$, indexed by double-indices $\left(i_{1}, i_{2}\right) \in\{0,1, \ldots, n\}^{2}$ by

$$
S_{\left(i_{1}, i_{2}\right)}:=\sum_{p=1}^{i_{1}} \sum_{q=1}^{i_{2}} \varepsilon_{(p, q)}
$$

and set $S_{\left(i_{1}, 0\right)} \equiv S_{\left(0, i_{2}\right)} \equiv 0$ for all $\left(i_{1}, i_{2}\right) \in\{0, \ldots, n\}^{2}$. Note that the following identity holds:

$$
\varepsilon_{\left(i_{1}, i_{2}\right)}=S_{\left(i_{1}, i_{2}\right)}-S_{\left(i_{1}-1, i_{2}\right)}+S_{\left(i_{1}-1, i_{2}-1\right)}-S_{\left(i_{1}, i_{2}-1\right)}
$$

i.e., the errors can be replaced by the respective "increments" on $[\mathbf{i}-\mathbf{1}, \mathbf{i}]=:\left[i_{1}-1, i_{1}\right] \times\left[i_{2}-1, i_{2}\right]$ of the 
partial sum process on the grid $\{0, \ldots, n\} \times\{0, \ldots, n\}$. We thus obtain

$$
Z_{n, 1}(\mathbf{x})=\frac{1}{\sigma\left\|\widetilde{K}_{\mathrm{B}, U}\right\| n \sqrt{\prod_{j=1}^{2} g_{j}\left(x_{j}\right) h_{j}}} \sum_{i, j=1}^{n} \widetilde{K}_{\mathrm{B}, U}\left(\frac{\mathbf{t}_{\left(i_{1}, i_{2}\right)}-\mathbf{x}}{\mathbf{h}}\right)\left[S_{\left(i_{1}, i_{2}\right)}-S_{\left(i_{1}-1, i_{2}\right)}+S_{\left(i_{1}-1, i_{2}-1\right)}-S_{\left(i_{1}, i_{2}-1\right)}\right]
$$

We can now re-sort the sum and obtain a sum that contains the increments of the function $\mathbf{z} \mapsto$ $\widetilde{K}_{\mathbf{B}, U}\left(\frac{G^{-1}(\mathbf{z})-\mathbf{x}}{\mathbf{h}}\right)$ instead of the increments of the partial sum process and obtain

$$
\begin{aligned}
Z_{n, 1}(\mathbf{x}) & =\frac{1}{\sigma\left\|\widetilde{K}_{\mathbf{B}, U}\right\| n \sqrt{\prod_{j=1}^{2} g_{j}\left(x_{j}\right) h_{j}}}\left\{\sum_{i_{1}, i_{2}=1}^{n-1} \Delta\left(\mathbf{z} \mapsto \widetilde{K}_{\mathbf{B}, U}\left(\frac{G^{-1}(\mathbf{z})-\mathbf{x}}{\mathbf{h}}\right) ;\left[\frac{\mathbf{i}-\mathbf{1}}{n+1}, \frac{\mathbf{i}}{n+1}\right]\right) S_{\left(i_{1}, i_{2}\right)}\right. \\
& -\sum_{i_{1}=1}^{n-1} \widetilde{K}_{\mathbf{B}, U}\left(\frac{\mathbf{t}_{\left(i_{1}+1, n\right)}-\mathbf{x}}{\mathbf{h}}\right) S_{\left(i_{1}, n\right)}+\sum_{i_{1}=1}^{n-1} \widetilde{K}_{\mathfrak{B}, U}\left(\frac{\mathbf{t}_{\left(i_{1}, n\right)}-\mathbf{x}}{\mathbf{h}}\right) S_{\left(i_{1}, n\right)} \\
& -\sum_{i_{2}=1}^{n-1} \widetilde{K}_{\mathbf{B}, U}\left(\frac{\mathbf{t}_{\left(n, i_{2}+1\right)}-\mathbf{x}}{\mathbf{h}}\right) S_{\left(n, i_{2}\right)}+\sum_{i_{2}=1}^{n-1} \widetilde{K}_{\mathbf{B}, U}\left(\frac{\mathbf{t}_{\left(n, i_{2}\right)}-\mathbf{x}}{\mathbf{h}}\right) S_{\left(n, i_{2}\right)} \\
& \left.+\Delta\left(\mathbf{z} \mapsto \widetilde{K}_{\mathfrak{B}, U}\left(\frac{G^{-1}(\mathbf{z})-\mathbf{x}}{\mathbf{h}}\right) S_{\left(z_{1}, z_{2}\right)} ;[0, n] \times[0, n]\right)\right\} .
\end{aligned}
$$

Observe that $\mathbf{x} \in[\delta, 1-\delta]^{2}, \mathbf{t}_{\left(i_{1}, n\right)}=\left(G_{1}^{-1}\left(i_{1} / n\right), 1\right), x_{\left(n, i_{2}\right)}=\left(1, G_{2}^{-1}\left(i_{2} / n\right)\right)$ and for large enough $n$, $\delta / h_{1} \wedge \delta / h_{2}>1$. From Assumption 1 and from $S_{\left(i_{1}, 0\right)} \equiv S_{\left(0, i_{2}\right)} \equiv 0$ for all $\left(i_{1}, i_{2}\right) \in\{0, \ldots, n\}^{2}$. It now follows that all terms except the first one in the latter representation of $Z_{n, 1}(\mathbf{x})$ are equal to zero for sufficiently large $n$, which implies

$$
Z_{n, 1}(\mathbf{x})=\frac{1}{\sigma\left\|\widetilde{K}_{\mathbf{B}, U}\right\| n \sqrt{\prod_{j=1}^{2} g_{j}\left(x_{j}\right) h_{j}}} \sum_{i_{1}, i_{2}=1}^{n-1} \Delta\left(\mathbf{z} \mapsto \widetilde{K}_{\mathcal{B}, U}\left(\frac{G^{-1}(\mathbf{z})-\mathbf{x}}{\mathbf{h}}\right) ;\left[\frac{\mathbf{i}-\mathbf{1}}{n+1}, \frac{\mathbf{i}}{n+1}\right]\right) S_{\left(i_{1}, i_{2}\right)} .
$$

for all $n \geq n_{0}$, for some $n_{0} \in \mathbb{N}$. This yields

$$
\begin{aligned}
& \left|Z_{n, 1}(\mathbf{x})-Z_{n, 2}(\mathbf{x})\right| \\
& =\frac{1}{\left\|\widetilde{K}_{\mathcal{B}, U}\right\| n \sqrt{\prod_{j=1}^{2} g_{j}\left(x_{j}\right) h_{j}}}\left|\sum_{i_{1}, i_{2}=1}^{n-1} \Delta\left(\mathbf{z} \mapsto \widetilde{K}_{\mathbf{B}, U}\left(\frac{G^{-1}(\mathbf{z})-\mathbf{x}}{\mathbf{h}}\right) ;\left[\frac{\mathbf{i}-\mathbf{1}}{n+1}, \frac{\mathbf{i}}{n+1}\right]\right)\left(\frac{S_{\left(i_{1}, i_{2}\right)}}{\sigma}-W\left(i_{1}, i_{2}\right)\right)\right| \\
& \leq \frac{\sup _{1 \leq i_{1}, i_{2} \leq n}\left|S_{\left(i_{1}, i_{2}\right)} / \sigma-W\left(i_{1}, i_{2}\right)\right|}{\left\|\widetilde{K}_{\mathbf{B}, U}\right\| n \sqrt{\prod_{j=1}^{2} g_{j}\left(x_{j}\right) h_{j}}} \sum_{i_{1}, i_{2}=1}^{n-1}\left|\Delta\left(\mathbf{z} \mapsto \widetilde{K}_{\mathbf{B}, U}\left(\frac{G^{-1}(\mathbf{z})-\mathbf{x}}{\mathbf{h}}\right) ;\left[\frac{\mathbf{i}-\mathbf{1}}{n+1}, \frac{\mathbf{i}}{n+1}\right]\right)\right| \\
& \leq \frac{\sup _{1 \leq\left(i_{1}, i_{2}\right) \leq n}\left|S_{\left(i_{1}, i_{2}\right)} / \sigma-W\left(i_{1}, i_{2}\right)\right|}{\left\|\widetilde{K}_{\mathfrak{B}, U}\right\| n \sqrt{\prod_{j=1}^{2} g_{j}\left(x_{j}\right) h_{j}}} \int_{\mathbb{R}^{2}}\left|D^{(1,1)} \widetilde{K}_{\mathfrak{B}, U}(\mathbf{z})\right| d \mathbf{z} .
\end{aligned}
$$


The assertion of the lemma now follows from Theorem 1 in Rio (1993), which gives the estimate

$$
\sup _{1 \leq i_{1}, i_{2} \leq n}\left|S_{\left(i_{1}, i_{2}\right)} / \sigma-W\left(i_{1}, i_{2}\right)\right|=O\left(n^{\frac{2-\delta}{2}} \sqrt{\log (n)}\right) \quad \text { a.s. }
$$

since, under the assumptions of Theorem $1, \mathbb{E}\left|\varepsilon_{(1,1)}\right|^{r}<\infty$ for $r>4 /(2-\delta)$. It follows that

$$
\sup _{\mathbf{x} \in[\delta, 1-\delta]^{2}}\left|Z_{n, 1}(\mathbf{x})-Z_{n, 2}(\mathbf{x})\right|=O\left(\sqrt{\frac{\log (n)}{h_{1} h_{2} n^{\delta}}}\right)=o\left((\log (n))^{-\frac{1}{2}}\right) .
$$

For the next approximation step we need that $1 /\left(n h_{1} h_{2}\right)=o\left(1 / \log (n)^{2}\right)$ whis is implied by the conditions of Theorem 1.

Lemma 4. Under the assumptions of Theorem 1 the process $Z_{n, 2}(\mathbf{x})$ can be approximated by $Z_{n, 3}(\mathbf{x})$ uniformly with respect to $\mathrm{x} \in[\delta, 1-\delta]^{2}$, i.e.

$$
\sup _{x \in[\delta, 1-\delta]^{2}}\left|Z_{n, 2}(x)-Z_{n, 3}(x)\right|=o\left(\log (n)^{-1 / 2}\right)
$$

Proof. There exists a number $n_{0} \in \mathbb{N}$ such that we obtain by integration by parts

$$
\int_{I} \widetilde{K}_{\mathbf{B}, U}\left(\frac{\mathbf{z}-\mathbf{x}}{\mathbf{h}}\right) d W(G(\mathbf{z}))=\int_{I} D^{(1,1)} \widetilde{K}_{\mathfrak{B}, U}\left(\frac{\mathbf{z}-\mathbf{x}}{\mathbf{h}}\right) W(G(\mathbf{z})) d \mathbf{z}
$$

for all $n \geq n_{0}$. Here, all terms obtained by integration by parts except the one on the right hand side vanish for sufficiently large $n$ since $W\left(z_{1}, 0\right) \equiv W\left(0, z_{2}\right) \equiv 0$ and all edge points lie outside the support of the kernel $\widetilde{K}_{\mathrm{B}, U}$. The increment of $\widetilde{K}_{\mathrm{B}, U}$ in the definition of the process $Z_{n, 2}$ can be expressed in terms of an integral as follows

$$
\Delta\left(\mathbf{z} \mapsto \widetilde{K}_{\mathcal{B}, U}\left(\frac{G^{-1}(\mathbf{z})-\mathbf{x}}{\mathbf{h}}\right) ;\left[\frac{\mathbf{i}-\mathbf{1}}{n+1}, \frac{\mathbf{i}}{n+1}\right]\right)=\int_{\left[\frac{\mathbf{i}-\mathbf{1}}{n+1}, \frac{\mathbf{i}}{n+1}\right]} D^{(1,1)}\left(\widetilde{K}_{\mathcal{B}, U}\left(\frac{G^{-1}(\mathbf{z})-\mathbf{x}}{\mathbf{h}}\right)\right) d \mathbf{z}
$$

see, e.g., Owen (2005), Section 9, where we used the notation

$$
D^{(1,1)}\left(\widetilde{K}_{\mathbf{B}, U}\left(\frac{G^{-1}(\mathbf{z})-\mathbf{x}}{\mathbf{h}}\right)\right)=D^{(1,1)}\left(\widetilde{K}_{\mathbf{B}, U}\right)\left(\frac{G^{-1}(\mathbf{z})-\mathbf{x}}{\mathbf{h}}\right) \cdot \frac{G_{1}^{-1^{\prime}}\left(z_{1}\right) G_{2}^{-1^{\prime}}\left(z_{2}\right)}{h_{1} h_{2}} .
$$


This gives

$$
\begin{aligned}
Z_{n, 2}(\mathbf{x}) & =\frac{1}{\left\|\widetilde{K}_{\mathbf{B}, U}\right\| n \sqrt{\prod_{j=1}^{2} g_{j}\left(x_{j}\right) h_{j}}} \sum_{i_{1}, i_{2}=0}^{n-1} \int_{\left[\frac{\mathbf{i}-\mathbf{1}}{n+1}, \frac{\mathbf{i}}{n+1}\right]} D^{(1,1)}\left(\widetilde{K}_{\mathbf{B}, U}\left(\frac{G^{-1}(\mathbf{z})-\mathbf{x}}{\mathbf{h}}\right)\right) d \mathbf{z} W\left(i_{1}, i_{2}\right) \\
& \stackrel{\mathcal{D}}{=} \frac{1}{\left\|\widetilde{K}_{\mathrm{B}, U}\right\| \sqrt{\prod_{j=1}^{2} g_{j}\left(x_{j}\right) h_{j}}} \sum_{i_{1}, i_{2}=0}^{n-1} \int_{\left[\frac{\mathbf{i}-\mathbf{1}}{n+1}, \frac{\mathbf{i}}{n+1}\right]} D^{(1,1)}\left(\widetilde{K}_{\mathbf{B}, U}\left(\frac{G^{-1}(\mathbf{z})-\mathbf{x}}{\mathbf{h}}\right)\right) d \mathbf{z} W\left(\frac{i_{1}}{n}, \frac{i_{2}}{n}\right) .
\end{aligned}
$$

By change of variables we further obtain

$$
\begin{aligned}
Z_{n, 2}(\mathbf{x}) & =\frac{1}{\left\|\widetilde{K}_{\mathrm{B}, U}\right\| \sqrt{\prod_{j=1}^{2} g_{j}\left(x_{j}\right) h_{j}}} \sum_{i_{1}, i_{2}=0}^{n-1} \int_{\left[\mathbf{t}_{\left(i_{1}-1, i_{2}-1\right)}, \mathbf{t}_{\left(i_{1}, i_{2}\right)}\right]} D^{(1,1)}\left(\widetilde{K}_{\mathbf{B}, U}\left(\frac{\mathbf{u}-\mathbf{x}}{\mathbf{h}}\right)\right) d \mathbf{u} W\left(\frac{i_{1}}{n}, \frac{i_{2}}{n}\right) \\
& =\frac{1}{\left\|\widetilde{K}_{\mathrm{B}, U}\right\| \sqrt{\prod_{j=1}^{2} g_{j}\left(x_{j}\right) h_{j}}} \sum_{i_{1}, i_{2}=0}^{n-1} \int_{\left[\mathbf{t}_{\left(i_{1}-1, i_{2}-1\right)}, \mathbf{t}_{\left(i_{1}, i_{2}\right)}\right]} D^{(1,1)}\left(\widetilde{K}_{\mathbf{B}, U}\left(\frac{\mathbf{u}-\mathbf{x}}{\mathbf{h}}\right)\right) d \mathbf{u} W\left(G_{1}\left(t_{i_{1}}\right), G_{2}\left(t_{i_{2}}\right)\right) .
\end{aligned}
$$

by definition of the design points. Moreover,

$$
\begin{aligned}
Z_{n, 3}(\mathbf{x}) & =\frac{1}{\left\|\widetilde{K}_{\mathbf{B}, U}\right\| \sqrt{\prod_{j=1}^{2} g_{j}\left(x_{j}\right) h_{j}}} \int_{I} D^{(1,1)}\left(\widetilde{K}_{\mathcal{B}, U}\left(\frac{\mathbf{u}-\mathbf{x}}{\mathbf{h}}\right)\right) W(G(\mathbf{u})) d \mathbf{u} \\
& =\frac{1}{\left\|\widetilde{K}_{\mathrm{B}, U}\right\| \sqrt{\prod_{j=1}^{2} g_{j}\left(x_{j}\right) h_{j}}} \sum_{i, j=1}^{n-1} \int_{\left[\mathbf{t}_{\left(i_{1}-1, i_{2}-1\right)}, \mathbf{t}_{\left(i_{1}, i_{2}\right)}\right]} D^{(1,1)}\left(\widetilde{K}_{\mathbf{B}, U}\left(\frac{\mathbf{u}-\mathbf{x}}{\mathbf{h}}\right)\right) W(G(\mathbf{u})) d \mathbf{u} .
\end{aligned}
$$

and hence

$$
\left|Z_{n, 2}(\mathbf{x})-Z_{n, 3}(\mathbf{x})\right|=\frac{1}{\left\|\widetilde{K}_{\mathrm{B}, U}\right\| \sqrt{h_{1} h_{2}}}\left|\sum_{i, j=1}^{n-1} \int_{\left[\mathbf{t}_{\mathbf{i}-\mathbf{1}}, \mathbf{t}_{\mathbf{i}}\right]} D^{(1,1)}\left(\widetilde{K}_{\mathbf{B}, U}\left(\frac{\mathbf{u}-\mathbf{x}}{\mathbf{h}}\right)\right)\left(W\left(G\left(\mathbf{t}_{\mathbf{i}}\right)\right)-W(G(\mathbf{u}))\right) d \mathbf{u}\right|
$$

Next we apply Theorem 3.2.1 in Khoshnevisan (2002) which gives a modulus of continuity for the Wiener sheet

$$
\left|W\left(G\left(\mathbf{t}_{\mathbf{i}}\right)\right)-W(G(\mathbf{z}))\right| \leq \sqrt{\frac{\log (n)}{n}} \sup _{k \geq n} \sup _{\substack{y, z \in I \\\|y-z\|_{\infty} \leq 1 / k}} \frac{|W(z)-W(y)|}{\sqrt{\frac{\log (n)}{n}}}=O\left(\sqrt{\log (n) n^{-1}}\right)
$$

almost surely. We also observe that

$$
\sum_{i, j=1}^{n-1} \int_{\left[\mathbf{t}_{\left(i_{1}-1, i_{2}-1\right)}, \mathbf{t}_{\left(i_{1}, i_{2}\right)}\right]}\left|D^{(1,1)}\left(\widetilde{K}_{\mathbf{B}, U}\left(\frac{\mathbf{u}-\mathbf{x}}{\mathbf{h}}\right)\right)\right| d \mathbf{u} \leq \int_{\mathbb{R}^{2}}\left|D^{(1,1)} \widetilde{K}_{\mathcal{B}, U}(z)\right| d z<\infty,
$$


which conclude the proof of this lemma.

\section{Lemma 5.}

$$
\left|Z_{n, 3}(\mathbf{x})-Z_{n, 5}(\mathbf{x})\right|=O_{P}\left(\log (n)\left(h_{1}+h_{2}\right)^{\frac{3}{2}} h_{1}^{-1 / 2} h_{2}^{-1 / 2}\right)
$$

Proof. Since $Z_{n, 3}$ and $Z_{n, 4}$ have the same probability structure we show that $\sup _{\mathbf{x} \in[\delta, 1-\delta]^{2}} \mid Z_{n, 4}(\mathbf{x})-$ $Z_{n, 5}(\mathbf{x}) \mid=O\left(\log (n) \frac{\left(h_{1}+h_{2}\right)^{\frac{3}{2}}}{\sqrt{h_{1} h_{2}}}\right)$ almost surely which proves the assertion of the lemma. Again, by integration by parts for sufficiently large $n$, since $\widetilde{K}_{\mathrm{B}, U}$ is of bounded support,

$$
Z_{n, 4}(\mathbf{x})-Z_{n, 5}(\mathbf{x})=\frac{1}{\left\|\widetilde{K}_{\mathbf{B}, U}\right\|} \int_{I} D^{(1,1)}\left[\widetilde{K}_{\mathbf{B}, U}\left(\frac{\mathbf{z}-\mathbf{x}}{\mathbf{h}}\right) \frac{\sqrt{g_{1}\left(z_{1}\right) g_{2}\left(z_{2}\right)}-\sqrt{g_{1}\left(x_{1}\right) g_{2}\left(x_{2}\right)}}{\sqrt{h_{1} h_{2} g_{1}\left(x_{1}\right) g_{2}\left(x_{2}\right)}}\right] W(\mathbf{z}) d \mathbf{z} .
$$

By change of variables, under Assumption 2 with the modulus of continuity of the Brownian sheet (here, $|u| \leq 1$, since $K$ has support contained in the cube $[-1,1] \times[-1,1])$

$$
\begin{aligned}
& Z_{n, 4}(\mathbf{x})-Z_{n, 5}(\mathbf{x}) \\
& =\frac{1}{\left\|\widetilde{K}_{\mathbf{B}, U}\right\|} \int_{\left[\frac{-\mathbf{x}}{\mathbf{h}}, \frac{1-\mathbf{x}}{\mathbf{h}}\right]} D^{(1,1)}\left[\widetilde{K}_{\mathbf{B}, U}(\mathbf{u}) \frac{\sqrt{g_{1}\left(x_{1}+u_{1} h_{1}\right) g_{2}\left(x_{2}+u_{2} h_{2}\right)}-\sqrt{g_{1}\left(x_{1}\right) g_{2}\left(x_{2}\right)}}{\sqrt{h_{1} h_{2} g_{1}\left(x_{1}\right) g_{2}\left(x_{2}\right)}}\right] W(\mathbf{x}+\mathbf{h u}) d \mathbf{u} \\
& =\frac{W(\mathbf{x})}{\left\|\widetilde{K}_{\mathbf{B}, U}\right\|} \int_{\left[\frac{-\mathbf{x}}{\mathbf{h}}, \frac{1-\mathbf{x}}{\mathbf{h}}\right]} D^{(1,1)}\left[\widetilde{K}_{\mathrm{B}, U}(\mathbf{u}) \frac{\sqrt{g_{1}\left(x_{1}+u_{1} h_{1}\right) g_{2}\left(x_{2}+u_{2} h_{2}\right)}-\sqrt{g_{1}\left(x_{1}\right) g_{2}\left(x_{2}\right)}}{\sqrt{h_{1} h_{2} g_{1}\left(x_{1}\right) g_{2}\left(x_{2}\right)}}\right] d \mathbf{u} \\
& +O\left(\log (n) \frac{\left(h_{1}+h_{2}\right)^{\frac{3}{2}}}{\sqrt{h_{1} h_{2}}}\right)
\end{aligned}
$$

almost surely. Furthermore,

$$
\begin{aligned}
& \int_{\left[\frac{-\mathbf{x}}{\mathbf{h}}, \frac{\mathbf{1 - x}}{\mathbf{h}}\right]} D^{(1,1)}\left[\widetilde{K}_{\mathbf{B}, U}(\mathbf{u}) \sqrt{g_{1}\left(x_{1}+u_{1} h_{1}\right) g_{2}\left(x_{2}+u_{2} h_{2}\right)}-\sqrt{g_{1}\left(x_{1}\right) g_{2}\left(x_{2}\right)}\right] d \mathbf{u} \\
& =\Delta\left(\mathbf{u} \mapsto \widetilde{K}_{\mathbf{B}, U}(\mathbf{u}) \sqrt{g_{1}\left(x_{1}+u_{1} h_{1}\right) g_{2}\left(x_{2}+u_{2} h_{2}\right)}-\sqrt{g_{1}\left(x_{1}\right) g_{2}\left(x_{2}\right)} ;\left[\frac{-\mathbf{x}}{\mathbf{h}}, \frac{\mathbf{1}-\mathbf{x}}{\mathbf{h}}\right]\right),
\end{aligned}
$$


which implies

$$
\begin{aligned}
Z_{n, 4}(\mathbf{x})-Z_{n, 5}(\mathbf{x})= & {\left[\widetilde{K}_{\mathbf{B}, U}\left(\frac{-x_{1}}{h_{1}}, \frac{-x_{2}}{h_{2}}\right)\left(\sqrt{g_{1}(0) g_{2}(0)}-\sqrt{g_{1}\left(x_{1}\right) g_{2}\left(x_{2}\right)}\right)\right.} \\
& -\widetilde{K}_{\mathbf{B}, U}\left(\frac{-x_{1}}{h_{1}}, \frac{1-x_{2}}{h_{2}}\right)\left(\sqrt{g_{1}(0) g_{2}(1)}-\sqrt{g_{1}\left(x_{1}\right) g_{2}\left(x_{2}\right)}\right) \\
& -\widetilde{K}_{\mathbf{B}, U}\left(\frac{1-x_{1}}{h_{1}}, \frac{-x_{2}}{h_{2}}\right)\left(\sqrt{g_{1}(1) g_{2}(0)}-\sqrt{g_{1}\left(x_{1}\right) g_{2}\left(x_{2}\right)}\right) \\
& \left.+\widetilde{K}_{\mathbf{B}, U}\left(\frac{1-x_{1}}{h_{1}}, \frac{1-x_{2}}{h_{2}}\right)\left(\sqrt{g_{1}(1) g_{2}(1)}-\sqrt{g_{1}\left(x_{1}\right) g_{2}\left(x_{2}\right)}\right)\right] \frac{W(\mathbf{x})}{\left\|\widetilde{K}_{\mathbf{B}, U}\right\| \sqrt{\prod_{j=1}^{2} g_{j}\left(x_{j}\right) h_{j}}} \\
& +O\left(\log (n) \frac{\left(h_{1}+h_{2}\right)^{\frac{3}{2}}}{\sqrt{h_{1} h_{2}}}\right) .
\end{aligned}
$$

For sufficiently large $n \in \mathbb{N}$ the first four summands vanish completely and thus

$$
\left|Z_{n, 4}(\mathbf{x})-Z_{n, 5}(\mathbf{x})\right|=O\left(\log (n)\left(h_{1}+h_{2}\right)^{\frac{3}{2}} / \sqrt{h_{1} h_{2}}\right),
$$

which completes the proof of this lemma.

Lemma 6. Under the assumptions of Theorem 1 the following result holds

$$
\sup _{\mathbf{x} \in[\delta, 1-\delta]^{2}}\left|Z_{n, 5}(\mathbf{x})\right| \stackrel{\mathcal{D}}{=} \sup _{\mathbf{x} \in \frac{1}{h_{1}}[\delta, 1-\delta] \times \frac{1}{h_{2}}[\delta, 1-\delta]}\left|Z_{n, 6}(\mathbf{x})\right|+o\left(\log (n)^{-1 / 2}\right)
$$

Proof. A combination of integration by parts, change of variables and the scaling property of the Brownian sheet yield

$$
Z_{n, 5}(\mathbf{x}) \stackrel{\mathcal{D}}{=} \frac{1}{\left\|\widetilde{K}_{\mathcal{B}, U}\right\| \sqrt{h_{1} h_{2}}} \int_{\left[0,1 / h_{1}\right] \times\left[0,1 / h_{2}\right]} D^{(1,1)} \widetilde{K}_{\mathcal{B}, U}\left(\mathbf{z}-\frac{\mathbf{x}}{\mathbf{h}}\right) W(\mathbf{z}) d \mathbf{z}
$$

With the definition of the sets $D_{<0}:=\left\{\left(z_{1}, z_{2}\right) \in \mathbb{R}^{2} \mid z_{1}<0 \vee z_{2}<0\right\}$ and $D_{>\frac{1}{h}}:=\left\{\left(z_{1}, z_{2}\right) \in \mathbb{R}^{2} \mid z_{1}>\right.$ $\left.1 / h_{1} \vee z_{2}>1 / h_{2}\right\}$ we obtain

$$
Z_{n, 6}\left(\frac{\mathbf{x}}{\mathbf{h}}\right)-Z_{n, 5}(\mathbf{x})=\frac{1}{\left\|\widetilde{K}_{\mathrm{B}, U}\right\|} \int_{D_{<0}} \widetilde{K}_{\mathrm{B}, U}\left(\mathbf{z}-\frac{\mathbf{x}}{\mathbf{h}}\right) d W(\mathbf{z})+\frac{1}{\left\|\widetilde{K}_{\mathcal{B}, U}\right\|} \int_{D_{>\frac{1}{h}}} \widetilde{K}_{\mathcal{B}, U}\left(\mathbf{z}-\frac{\mathbf{x}}{\mathbf{h}}\right) d W(\mathbf{z})
$$

For $\mathbf{z} \in D_{>\frac{1}{h}}, \mathbf{x} \in[\delta, 1-\delta]^{2}$ we further have $z_{j}-x_{j} / h_{j}>\delta / h_{j} \quad$ for $\quad j=1 \vee j=2$ and for $\mathbf{z} \in D_{<0}, \mathbf{x} \in$ $[\delta, 1-\delta]^{2}$ we obtain $z_{j}-x_{j} / h_{j}<-\delta / h_{j}$ for $\quad j=1 \vee j=2$. Since $\delta$ is a fixed positive constant there 
exists a number $n_{0} \in \mathbb{N}$ such that $\widetilde{K}_{\mathcal{B}, U}(\mathbf{z}-\mathbf{x} / \mathbf{h}) \equiv 0$ for all $\mathbf{z} \in D_{<0} \cup D_{>\frac{1}{h}}, \mathbf{x} \in[\delta, 1-\delta]^{2}$. Hence, for $n \geq n_{0} Z_{n, 6}(\mathbf{x} / \mathbf{h}) \stackrel{\mathcal{D}}{=} Z_{n, 5}(\mathbf{x})$ and

$$
\sup _{\mathbf{x} \in[\delta, 1-\delta]^{2}}\left|Z_{n, 6}(\mathbf{x} / \mathbf{h})\right| \stackrel{\mathcal{D}}{=} \sup _{\mathbf{x} \in[\delta, 1-\delta]^{2}}\left|Z_{n, 5}(\mathbf{x})\right| \stackrel{\mathcal{D}}{=} \sup _{\mathbf{x} \in \frac{1}{h_{1}}[\delta, 1-\delta] \times \frac{1}{h_{2}}[\delta, 1-\delta]}\left|Z_{n, 6}(\mathbf{x})\right|,
$$

which completes the proof of the lemma.

\section{Proof of Theorem 1}

Given the assumptions of Theorem 1 regarding the relative growth of the bandwidths $h_{1}$ and $h_{2}$, the system of sets $\left\{\frac{1}{h_{1}}[\delta, 1-\delta] \times \frac{1}{h_{2}}[\delta, 1-\delta] \mid n \in \mathbb{N}\right\}$ with volumes $(1-2 \delta)^{2} /\left(h_{1} h_{2}\right)$ is a blowing up system of sets according to Definition 14.1 in Piterbarg (1996). An application of Theorem 14.3 therein thus yields

$$
\lim _{n \rightarrow \infty} \mathbb{P}\left(\sup _{\mathbf{x} \in \frac{1}{h_{1}}[\delta, 1-\delta] \times \frac{1}{h_{2}}[\delta, 1-\delta]}\left(\left|Z_{n, 6}(\mathbf{x})\right|-l_{n}\right) l_{n}<\kappa\right)=e^{-2 e^{-\kappa}}
$$

The following lemma provides the last missing piece, the negligibility of the remainder $R_{n, 0}$.

Lemma 7. Let Assumption 1 and 2 be satisfied. Then

$$
\sup _{\mathbf{x} \in[\delta, 1-\delta]}\left|R_{n, 0}(\mathbf{x})\right|=O_{P}\left(\sqrt{\log (n)}\left(h_{1}+h_{2}\right)\right)
$$

Proof. Lemma 1 implies the decomposition $\mathbf{B}_{n, \mathbf{x}}^{-1}=\mathbf{B}_{\mathbf{x}}^{-1}+\mathbf{R}_{n, \mathbf{x}}^{-}$with a $(k+1)(k+2) / 2 \times(k+1)(k+2) / 2$ matrix $\mathrm{R}_{n, \mathbf{x}}^{-}$that has the property

$$
\begin{aligned}
\sup _{\mathbf{x} \in[\delta, 1-\delta]^{2}}\left|\left(R_{\mathbf{B}_{n, \mathbf{x}}}^{-}\right)_{p, q}\right|=O\left(h_{1}+h_{2}\right), \quad(p, q) \in\{1, \ldots,(k+1)(k+2) / 2\}^{2} . \\
R_{n, 0}(\mathbf{x})=\frac{\sqrt{g_{1}\left(x_{1}\right) g_{2}\left(x_{2}\right)}}{\sigma n \sqrt{h_{1} h_{2}}} \sum_{i_{1}, i_{2}=1}^{n} \widetilde{K}_{\mathbf{R}_{n, x}, U}\left(\frac{\mathbf{t}_{\mathbf{i}}-\mathbf{x}}{\mathbf{h}}\right) \varepsilon_{\left(i_{1}, i_{2}\right)} \\
=\frac{\sqrt{g_{1}\left(x_{1}\right) g_{2}\left(x_{2}\right)}}{\sigma n \sqrt{h_{1} h_{2}}} \sum_{i_{1}, i_{2}=1}^{n} U^{T}(\mathbf{0}) \mathbf{R}_{n, x}^{-} U\left(\frac{\mathbf{t}_{\mathbf{i}}-\mathbf{x}}{\mathbf{h}}\right) K\left(\frac{\mathbf{t}_{\mathbf{i}}-\mathbf{x}}{\mathbf{h}}\right) \varepsilon_{\left(i_{1}, i_{2}\right)} .
\end{aligned}
$$

The quantity $U^{T}(\mathbf{0}) \mathbf{R}_{n, x}^{-}=\left(\left(r_{n, \mathbf{x}}^{-}\right)_{1,1}, \ldots,\left(r_{n, \mathbf{x}}^{-}\right)_{1,(k+1)(k+2) / 2}\right) \in \mathbb{R}^{1 \times(k+1)(k+2) / 2}$ is the first row of the 
$\operatorname{matrix} \mathbf{R}_{n, x}^{-}$and $U\left(\frac{\mathbf{t}_{\mathbf{i}}-\mathbf{x}}{\mathbf{h}}\right)=\left(U^{(1)}\left(\frac{\mathbf{t}_{\mathbf{i}}-\mathbf{x}}{\mathbf{h}}\right), \ldots, U^{((k+1)(k+2) / 2)}\left(\frac{\mathbf{t}_{\mathbf{i}}-\mathbf{x}}{\mathbf{h}}\right)\right) \in \mathbb{R}^{(k+1)(k+2) / 2}$, hence we can write

$$
\sum_{p=1}^{(k+1)(k+2) / 2}\left(r_{n, \mathbf{x}}^{-}\right)_{1, p} \frac{\sqrt{g_{1}\left(x_{1}\right) g_{2}\left(x_{2}\right)}}{\sigma n \sqrt{h_{1} h_{2}}} \sum_{i_{1}, i_{2}=1}^{n} U^{(p)}\left(\frac{\mathbf{t}_{\mathbf{i}}-\mathbf{x}}{\mathbf{h}}\right) K\left(\frac{\mathbf{t}_{\mathbf{i}}-\mathbf{x}}{\mathbf{h}}\right) \varepsilon_{\left(i_{1}, i_{2}\right)} .
$$

For each fixed number $p_{0} \in\{1, \ldots,(k+1)(k+2) / 2\}$ we find

$$
\begin{aligned}
& \sup _{\mathbf{x} \in[\delta, 1-\delta]^{2}}\left|\frac{\left(r_{n, \mathbf{x}}^{-}\right)_{1, p_{0}} \sqrt{g_{1}\left(x_{1}\right) g_{2}\left(x_{2}\right)}}{\sigma n \sqrt{h_{1} h_{2}}} \sum_{i_{1}, i_{2}=1}^{n} U^{\left(p_{0}\right)}\left(\frac{\mathbf{t}_{\mathbf{i}}-\mathbf{x}}{\mathbf{h}}\right) K\left(\frac{\mathbf{t}_{\mathbf{i}}-\mathbf{x}}{\mathbf{h}}\right) \varepsilon_{\left(i_{1}, i_{2}\right)}\right| \\
& \leq \sup _{\mathbf{x} \in[\delta, 1-\delta]^{2}}\left|\left(r_{n, \mathbf{x}}^{-}\right)_{1, p_{0}} \sqrt{g_{1}\left(x_{1}\right) g_{2}\left(x_{2}\right)}\right| \sup _{\mathbf{x} \in[\delta, 1-\delta]^{2}}\left|\frac{1}{\sigma n \sqrt{h_{1} h_{2}}} \sum_{i_{1}, i_{2}=1}^{n} U^{\left(p_{0}\right)}\left(\frac{\mathbf{t}_{\mathbf{i}}-\mathbf{x}}{\mathbf{h}}\right) K\left(\frac{\mathbf{t}_{\mathbf{i}}-\mathbf{x}}{\mathbf{h}}\right) \varepsilon_{\left(i_{1}, i_{2}\right)}\right| \\
& =\sup _{\mathbf{x} \in[\delta, 1-\delta]^{2}}\left|\left(r_{n, \mathbf{x}}^{-}\right)_{1, p_{0}} \sqrt{g_{1}\left(x_{1}\right) g_{2}\left(x_{2}\right)}\right| O_{P}(\sqrt{\log (n)})=O_{P}\left(\left(h_{1}+h_{2}\right) \sqrt{\log (n)}\right)
\end{aligned}
$$

with the same arguments as used before to prove the convergence of $\sup _{\mathbf{x} \in[\delta, 1-\delta]^{2}}\left|Z_{n, 1}(x)\right|$, property (12) and since the design densities $g_{1}$ and $g_{2}$ are bounded.

An application of lemmas 3-7 completes the proof of Theorem 1.

\section{Proof of Corollary 1}

In order to prove the corollary we need to calculate the bias of the estimator $\hat{f}_{n}(\mathbf{x})$. For this matter we need the following auxiliary result.

Lemma 8. If Assumptions 1 and 2 hold the matrix $B_{\mathbf{x}}$ is positive definite.

Proof. Let $\mathbf{v} \in \mathbb{R}^{(k+1)(k+2) / 2} \backslash\{\mathbf{0}\}$. Assumption 1 implies that

$$
\mathbf{v}^{T} \mathbf{B} \mathbf{v}=\iint\left(\mathbf{v}^{T} U(\mathbf{u})\right)^{2} K(\mathbf{u}) d \mathbf{u} \geq K_{1} \int_{-D}^{D} \int_{-D}^{D}\left(\mathbf{v}^{T} U(\mathbf{u})\right)^{2} d \mathbf{u} \geq 0
$$

For $\mathbf{v} \neq \mathbf{0}$ the quantity $\mathbf{v}^{T} U(\mathbf{u}) \neq 0$ is a plolynomial in $\mathbf{u}$ of degree $\leq k$ and it can only be equal to zero at a finite number of points. Since also $D>0$ it follows that $\mathbf{v}^{T} \mathbf{B v}>0$ and since the design densities $g_{1}$ and $g_{2}$ are bounded away from zero it also follows that $\mathbf{v}^{T} \mathbf{B}_{\mathbf{x}} \mathbf{v}=g_{1}\left(x_{1}\right) g_{2}\left(x_{2}\right) \mathbf{v}^{T} \mathbf{B} \mathbf{v}>0$, which concludes the proof of the lemma. 
Corollary 3. For sufficiently large $n \in \mathbb{N}$ the matrix $\boldsymbol{B}_{n, \mathbf{x}}$ is positive definite, i.e., there exists a positive constant $\lambda_{0}$ and a positive number $n_{0} \in \mathbb{N}$ such that the smallest eigenvalue $\lambda_{\min }\left(\mathbb{B}_{n, \mathbf{x}}\right) \geq \lambda_{0}$, for all $\mathbf{x} \in[0,1]^{2}$ if $n \geq n_{0}$.

Also, we make use of the fact that the local polynomial estimator of order $k$ reproduces polynomials of degree $\leq k$. This means that for any polynomial $Q$ with $Q(\mathbf{x})=\sum_{\boldsymbol{\beta} \in\{0, \ldots, k\}^{2},|\boldsymbol{\beta}| \leq k} a_{\boldsymbol{\beta}} \mathbf{x}^{\boldsymbol{\beta}}$ for $\mathbf{x} \in \mathbb{R}^{2}$ the following equality holds

$$
\sum_{i_{1}, i_{2}=1}^{n} Q\left(\mathbf{t}_{\left(i_{1}, i_{2}\right)}\right) W_{n,\left(i_{1}, i_{2}\right)}(\mathbf{x})=Q(\mathbf{x})
$$

and hence

$$
\operatorname{bias}\left(\hat{f}_{n}, f, \mathbf{x}\right)=\mathbb{E} \hat{f}_{n}(\mathbf{x})-f(\mathbf{x})=\sum_{i_{1}, i_{2}=1}^{n}\left[f\left(\mathbf{t}_{\left(i_{1}, i_{2}\right)}\right)-f(\mathbf{x})\right] W_{n,\left(i_{1}, i_{2}\right)}(\mathbf{x})
$$

since (14) implies the identity $\sum_{i_{1}, i_{2}=1}^{n} W_{\left.n, i_{1}, i_{2}\right)}(\mathbf{x})=1$.

Equation (14) further implies that $\sum_{i_{1}, i_{2}=1}^{n}\left(\mathbf{t}_{\left(i_{1}, i_{2}\right)}-\mathbf{x}\right)^{\beta} W_{n,\left(i_{1}, i_{2}\right)}(\mathbf{x})=0$ for all multi-indices $\beta \in$ $\{0, \ldots, k\}^{2},|\beta| \leq k$. By Taylor expansion and from (3) we obtain

$$
\begin{aligned}
\operatorname{bias}\left(\hat{f}_{n}, f, \mathbf{x}\right) & \leq \sum_{i_{1}, i_{2}=1}^{n} C_{f, k}\left\|\mathbf{t}_{\left(i_{1}, i_{2}\right)}-\mathbf{x}\right\|^{a}\left|W_{n,\left(i_{1}, i_{2}\right)}(\mathbf{x})\right| \leq C_{f, k}\left(h_{1}+h_{2}\right)^{k+a} \sum_{i_{1}, i_{2}=1}^{n}\left\|\mathbf{t}_{\left(i_{1}, i_{2}\right)}-\mathbf{x}\right\|^{a}\left|W_{n,\left(i_{1}, i_{2}\right)}(\mathbf{x})\right| \\
& \leq \frac{K_{2} C_{f, k}\left(h_{1}+h_{2}\right)^{k+a}}{\lambda_{\min } n^{2} h_{1} h_{2}} \sum_{i_{1}, i_{2}=1}^{n}\left\|U\left(\frac{\mathbf{t}_{\left(i_{1}, i_{2}\right)}-\mathbf{x}}{\mathbf{h}}\right)\right\| I_{\left[x_{1}-h_{1}, x_{1}+h_{1}\right]}\left(t_{i_{1}}\right) I_{\left[x_{2}-h_{2}, x_{2}+h_{2}\right]}\left(t_{i_{2}}\right) .
\end{aligned}
$$

Since $t_{i_{j}}=G^{-1}\left(i_{j} /(n+1)\right)$ and $G$ is strictly increasing the indicator functions $I_{\left[x_{j}-h_{j}, x_{j}+h_{j}\right]}\left(t_{i_{j}}\right)$ can be replaced by $I_{\left[G_{j}\left(x_{j}-h_{j}\right), G_{j}\left(x_{j}+h_{j}\right)\right]}\left(G_{j}\left(t_{i_{j}}\right)\right)$ for $j=1$ and $j=2$. This finally implies

$$
\operatorname{bias}\left(\hat{f}_{n}, f, \mathbf{x}\right)=O\left(\left(h_{1}+h_{2}\right)^{k+a}\right)
$$




\section{Proofs of Theorem 2 and Corollary 2}

In this section we sketch the extension of the proofs of the results of Section 6.2 to the case of general dimension $d$. Here, we need a multivariate generalization of the concept of functions of bounded variation for which we make use of the elementary, intuitive approach in terms of suitable generalizations of increments such as it is given in Owen (2005). The generalization of the concept of increments $\Delta_{d}$ of a function $f$ over $d$-dimensional intervals $[\mathbf{a}, \mathbf{b}]$ that is relevant for us in this context is given by the definition

$$
\Delta_{d}(f ;[\mathbf{a}, \mathbf{b}]):=\sum_{\boldsymbol{\alpha} \in\{0,1\}^{d}}(-1)^{|\boldsymbol{\alpha}|} f(\mathbf{b}+\boldsymbol{\alpha} \odot(\mathbf{a}-\mathbf{b}))
$$

where $\boldsymbol{\alpha} \odot(\mathbf{a}-\mathbf{b})=\left(\alpha_{1} \cdot\left(a_{1}-b_{1}\right), \ldots, \alpha_{d} \cdot\left(a_{d}-b_{d}\right)\right)^{T}$ denotes the vector of component-wise products of the multi-index $\boldsymbol{\alpha}$ and the vector $\mathbf{b}-\mathbf{a}$. The above defined increments $\Delta_{d}$ of a function $f$ over $d$-dimensional intervals $[\mathbf{a}, \mathbf{b}]$ have the following property

$$
\Delta_{d}(f ;[\mathbf{a}, \mathbf{b}])=\int_{[\mathbf{a}, \mathbf{b}]} D^{(1, \ldots, 1)} f(\mathbf{x}) d \mathbf{x}
$$

Lemma 3d. There exists a Wiener sheet $W$ on a suitable probability space such that

$$
\sup _{x \in[\delta, 1-\delta]^{d}}\left|Z_{n, 1, d}(\mathbf{x})-Z_{n, 2, d}(\mathbf{x})\right|=O\left(\frac{\log (n)}{n^{\delta} \sqrt{h_{1} h_{2}}}\right)
$$

where

$$
Z_{n, 1, d}(\mathbf{x}):=\frac{1}{\sigma\left\|\widetilde{K}_{\mathbf{B}, U}\right\| n^{\frac{d}{2}} \sqrt{\prod_{j=1}^{d} g_{j}\left(x_{j}\right) h_{j}}} \sum_{i_{1}, \ldots, i_{d}=1}^{n} \widetilde{K}_{\mathbf{B}, U}\left(\frac{\mathbf{t}_{\mathbf{i}}-\mathbf{x}}{\mathbf{h}}\right) \varepsilon_{i_{1}, \ldots, i_{d}}
$$

and

$Z_{n, 2, d}(\mathbf{x}):=\frac{1}{\left\|\widetilde{K}_{\mathfrak{B}, U}\right\| n^{\frac{d}{2}} \sqrt{\prod_{j=1}^{d} g_{j}\left(x_{j}\right) h_{j}}} \sum_{i_{1}, \ldots, i_{d}=1}^{n} \Delta_{d}\left(\mathbf{z} \mapsto \widetilde{K}_{\mathbf{B}, U}\left(\frac{G^{-1}(\mathbf{z})-\mathbf{x}}{\mathbf{h}}\right) ;\left[\frac{\mathbf{i}-\mathbf{1}}{n+1}, \frac{\mathbf{i}}{n+1}\right]\right) W\left(i_{1}, \ldots, i_{2}\right)$

Proof. For general dimension $d$ define the partial sum $S_{\left(i_{1}, \ldots, i_{d}\right)}:=\sum_{p_{1}}^{i_{1}} \ldots \sum_{p_{d}=1}^{i_{d}} \varepsilon_{\left(p_{1}, \ldots, p_{d}\right)}$ and set $S_{\mathbf{i}} \equiv 0$ if $i_{j}=0$ for at least one $j \in\{1, \ldots, d\}$. Again we can replace the errors by suitable increments of the 
partial sum $S_{(\cdot)}$ over $[\mathbf{i}-\mathbf{1}, \mathbf{i}]$ :

$$
\varepsilon_{\left(i_{1}, \ldots, i_{d}\right)}=\Delta_{d}\left(S_{(\cdot)},[\mathbf{i}-\mathbf{1}, \mathbf{i}]\right)=\sum_{\boldsymbol{\alpha} \in\{0,1\}^{d}}(-1)^{|\boldsymbol{\alpha}|} S_{(\mathbf{i}-\boldsymbol{\alpha})}=\sum_{\boldsymbol{\alpha} \in\{0,1\}^{d}}(-1)^{|\boldsymbol{\alpha}|} S_{\left(i_{1}-\alpha_{1}, \ldots, i_{d}-\alpha_{d}\right)}
$$

With the same arguments as in the two dimensional case the replacement of the errors by the increments of the partial sums yields for sufficiently large $n$ (such that all boundary terms vanish)

$$
Z_{n, 1, d}(\mathbf{x})=\frac{1}{\sigma\left\|\widetilde{K}_{\mathrm{B}, U}\right\| n^{\frac{d}{2}} \prod_{j=1}^{d} \sqrt{h_{i_{j}} g_{i_{j}}\left(x_{1}\right)}} \sum_{i_{1}, \ldots, i_{d}=1}^{n-1} \Delta_{d}\left(\mathbf{z} \mapsto \widetilde{K}_{\mathrm{B}, U}\left(\frac{G^{-1}(\mathbf{z})-\mathbf{x}}{\mathbf{h}}\right) ;\left[\frac{\mathbf{i}-\mathbf{1}}{n+1}, \frac{\mathbf{i}}{n+1}\right]\right) S_{i_{1}, \ldots, i_{d}} .
$$

Another application of Theorem 1 in Rio (1993) yields the estimate

$$
\sup _{x \in[\delta, 1-\delta]^{d}}\left|Z_{n, 1, d}(\mathbf{x})-Z_{n, 2, d}(\mathbf{x})\right|=O\left(\left(\frac{n^{\delta-d} \log (n)}{n^{d} h_{1} \cdot \ldots \cdot h_{d}}\right)^{\frac{1}{2}}\right)=O\left(\left(\frac{\log (n)}{n^{\delta} h_{1} \cdot \ldots \cdot h_{d}}\right)^{\frac{1}{2}}\right) .
$$

Lemma $4 \mathbf{d}$. Under the assumptions of Theorem 2 the process $Z_{n, 2}(\mathbf{x})$ can be approximated by $Z_{n, 3}(\mathbf{x})$ uniformly with respect to $\mathrm{x} \in[\delta, 1-\delta]^{d}$, i.e.

$$
\sup _{\mathbf{x} \in[\delta, 1-\delta]^{d}}\left|Z_{n, 2, d}(\mathbf{x})-Z_{n, 3, d}(\mathbf{x})\right|=o\left(\frac{1}{\sqrt{\log (n)}}\right)
$$

where

$$
Z_{n, 3}(\mathbf{x}):=\frac{1}{\left\|\widetilde{K}_{\mathrm{B}, U}\right\| \prod_{j=1}^{d} \sqrt{h_{j} g_{j}\left(x_{j}\right)}} \int_{I} \widetilde{K}_{\mathrm{B}, U}\left(\frac{\mathbf{z}-\mathbf{x}}{\mathbf{h}}\right) d W(G(\mathbf{z}))
$$

Proof. Also for the $d$-dimensional case there exists a number $n_{0} \in \mathbb{N}$ such that we obtain by integration by parts for all $n \geq n_{0}$

$$
\int_{I} \widetilde{K}_{\mathfrak{B}, U}\left(\frac{\mathbf{z}-\mathbf{x}}{\mathbf{h}}\right) d W(G(\mathbf{z}))=\int_{I} D^{(1,1)} \widetilde{K}_{\mathfrak{B}, U}\left(\frac{\mathbf{z}-\mathbf{x}}{\mathbf{h}}\right) W(\mathbf{z}) d \mathbf{z}
$$

i.e., all boundary terms vanish for sufficiently large $n$. To prove the assertion of the lemma we can now 
use equation (15) and follow the lines of the proof of Lemma 4 and obtain the estimate

$$
\sup _{\mathbf{x} \in[\delta, 1-\delta]^{d}}\left|Z_{n, 2, d}(\mathbf{x})-Z_{n, 3, d}(\mathbf{x})\right|=O_{P}\left(\sqrt{\log (n)} / \sqrt{n h_{1} \cdot \ldots \cdot h_{d}}\right) .
$$

Lemma 5d. Assume that the assumptions of Theorem 2 hold. Then

$$
\left|Z_{n, 3}(\mathbf{x})-Z_{n, 5}(\mathbf{x})\right|=O_{P}\left(\log (n)\left(h_{1}+\ldots+h_{d}\right)^{\frac{(2 d-1) \vee 3}{2}} / \sqrt{h_{1} \cdot \ldots \cdot h_{d}}\right),
$$

where

$$
Z_{n, 5, d}(\mathbf{x}):=\frac{1}{\left\|\widetilde{K}_{\mathrm{B}, U}\right\| \sqrt{h_{1} \cdot \ldots \cdot h_{d}}} \int_{I} \widetilde{K}_{\mathrm{B}, U}\left(\frac{\mathbf{z}-\mathbf{x}}{\mathbf{h}}\right) d W(\mathbf{z}) .
$$

Proof. Again we make an intermediate step by introducing a further process, $Z_{n, 4, d}$, that has the same probability structure as $Z_{n, 3, d}$ and define

$$
Z_{n, 4, d}(\mathbf{x}):=\frac{1}{\left\|\widetilde{K}_{\mathrm{B}, U}\right\| \sqrt{\prod_{j=1}^{d} h_{j} g_{j}}} \int_{I} \widetilde{K}_{\mathrm{B}, U}\left(\frac{\mathbf{z}-\mathbf{x}}{\mathbf{h}}\right) \sqrt{g_{1}\left(z_{1}\right) \cdot \ldots \cdot g_{d}\left(z_{d}\right)} d W(\mathbf{z}) .
$$

By assumption, the design densities $g_{j}$ are continuously diffeentiable up to order $(d-1) \vee 1, j=1, \ldots, d$. By higher order Taylor expansion of the difference $\sqrt{\prod_{j=1}^{d} g_{j}\left(x_{j}+u_{j} h_{j}\right)}-\sqrt{\prod_{j=1}^{d} g_{j}\left(x_{j}\right)}$ with the same arguments applied in the proof of Lemma 5 we obtain the estimate

$$
\left|Z_{n, 3, d}(\mathbf{x})-Z_{n, 5, d}(\mathbf{x})\right|=\left(\log (n)\left(h_{1}+\ldots+h_{d} \frac{(2 d-1) \vee 3}{2} / \sqrt{h_{1} \cdot \ldots \cdot h_{d}}\right),\right.
$$

which holds almost surely and uniformly in $\mathbf{x} \in[\delta, 1-\delta]^{d}$.

The generalization of Lemma 6 and Lemma 7 and both proofs, as well as the further steps in the proofs of Theorem 1 and Corollary 2 are straightforward and are therefore omitted. 

